\title{
Matching-invariant running quark masses in Quantum Chromodynamics
}

\author{
H. M. Chen, ${ }^{1}$ L. M. Liu,${ }^{1}$ J. T. Wang, ${ }^{1}$ M. Waqas,${ }^{1}$ G. X. Peng,,${ }^{1,2,3}$ \\ ${ }^{1}$ School of Physics \& College of Nuclear Science and Technology, \\ University of Chinese Academy of Sciences, Beijing 100049, China \\ ${ }^{2}$ Synergetic Innovation Center for Quantum Effects and Applications \\ Hunan Normal University, Changsha 410081, China \\ ${ }^{3}$ Theoretical Physics Center for Science Facilities, Institute of High Energy Physics, Beijing 100049, China
}

\begin{abstract}
The renormalization group equations of the QCD running coupling and quark masses are solved in a mathematically strict way. General relations between the standard expansion coefficients and the beta and gamma functions have been accordingly deduced. It is thus found that previous truncated expressions either loses an infinite number of known logarithmic terms, which can be given in a closed form, or includes a large number of incorrect terms. Consequently, a new expansion given by Peng with the fastest convergence speed is adopted. Given the matching-invariant beta and gamma functions to four-loop level, the scale parameters for the coupling and masses are calculated from the new expressions and the latest experimental average of $\alpha_{s}\left(M_{\mathrm{Z}}\right)$, for the order from 1 to 4 and the number of flavors from 3 to 6 . In addition, the conventional quark mass is not continuous at thresholds. In this paper, we derive matching-invariant quark masses which are continuous everywhere. They are expanded as an obvious function of the logarithmic $\Lambda$ scaled energy. The expansion coefficients are related to the original $\gamma$ and $\beta$ functions, with concretization to four loop level. The results show that the new expressions for the quark masses converge indeed much faster.
\end{abstract}

PACS numbers: 21.65.+f, 24.85.+p, 12.38.-t, 11.30.Rd

\section{INTRODUCTION}

As is well known, the coupling and quark masses in Quantum Chronmodynamics (QCD) [1] are running, i.e., they depend on the renormalization point. To have full thermodynamic consistency in practical applications [2, $3]$, it is necessary to include the renormalization-group (RG) dependence of the coupling and masses.

In nuclear and particle physics, it is usually convenient to have an explicit expression of the coupling as a function of the renormalization point $u$. The standard approach is to expand it to a series of $1 / \ln \left(u^{2} / \Lambda^{2}\right)$, where $\Lambda$ is a QCD scale parameter and customarily called $\Lambda_{\mathrm{QCD}}$. Another approach to calculating the coupling at a given $u$ is to truncate the beta series in the RG equation, and then solve the corresponding truncated differential equation numerically. The relation between the standard expansion coefficients and the beta function can be found to order 3 in Ref. [4], and to order 4 in Ref. [5].

In the standard minimum subtraction scheme [6] or its modified version [7] ( $\overline{\mathrm{MS}})$, the QCD coupling is, in principle, not continuous at the quark thresholds, which is surely inconvenient for applications. However, a new matching-invariant coupling can be given by absorbing loop-effects into the $\overline{\mathrm{MS}}$ definition [8]. For this the beta function should be linearly recombined. The general relation between the new and original beta coefficients will be discussed, with concrete expressions to 4-loop level. The same thing is also true for quark running masses. But, instead of changing the beta function, one should modify the gamma function.

The running quark masses are conventionally expressed as a series of the running coupling. Because the relation between the coupling and the renormalization point is not exactly known, the conventional expression causes indirect imprecision.

The paper is organized as follows. In Sec. II, the RG equation for the coupling is solved in a mathematically strict way, and the general relation between the standard expansion coefficients and the beta function is accordingly obtained. In Sec. III, the new expression given by Peng is compared with the conventional approaches to show that the new expression converges much faster [8]. Section IV and B works with the matching condition of the coupling. In addition, a matching-invariant coupling is derived in Sec. C. The relation between the corresponding new and original beta and gamma functions is deduced. Section V is dedicated to the quark running masses. The RG equation for the masses is solved mathematically with the corresponding gamma function for the matching-invariant masses given to order 4 . The quark masses are expressed first as a function of the coupling, then expanded to a direct series of the logarithmic renormalization point. Comparison with the conventional expansion and the truncation in the RG equation show that the new expression has the fastest convergence speed. Finally a summary is presented in Sec. VI.

\section{SOLUTION OF THE RG EQUATION FOR THE COUPLING}

The renormalization-group equation for the QCD running coupling $\alpha=\alpha_{s} / \pi=g^{2} /\left(4 \pi^{2}\right)$ is

$$
\frac{\mathrm{d} \alpha}{\mathrm{d} \ln u^{2}}=-\sum_{i=0}^{\infty} \beta_{i} \alpha^{i+2} \equiv \beta(\alpha)
$$


where the beta function was calculated, in $\overline{\mathrm{MS}}$, to oneloop level in Ref. [9], to two-loop in Ref. [10], to threeloop in Ref. [11] and to four-loop in Ref. [12]. The beta coefficients $\beta_{i}$ can be expressed as polynomials of the number of flavors $N_{\mathrm{f}}$, i.e.,

$$
\left[\begin{array}{l}
\beta_{0} \\
\beta_{1} \\
\beta_{2} \\
\beta_{3}
\end{array}\right]=\left[\begin{array}{cccc}
11 / 4 & -1 / 6 & 0 & 0 \\
51 / 8 & -19 / 24 & 0 & 0 \\
\frac{2857}{128} & -\frac{5033}{1152} & \frac{325}{3456} & 0 \\
\beta_{3,0} & \beta_{3,1} & \beta_{3,2} & \frac{1093}{186624}
\end{array}\right]\left[\begin{array}{c}
1 \\
N_{\mathrm{f}} \\
N_{\mathrm{f}}^{2} \\
N_{\mathrm{f}}^{3}
\end{array}\right]
$$

with $\beta_{3,0}=(891 / 64) \zeta_{3}+149753 / 1536 \approx$ $114.2303287, \beta_{3,1}=-(1627 / 1728) \zeta_{3}-1078361 / 41472 \approx$ $-27.13394382, \beta_{3,2}=(809 / 2592) \zeta_{3}+50065 / 41472 \approx$ 1.582379064, where $\zeta$ is the Riemann zeta function, and $\zeta_{2}=\pi^{2} / 6, \zeta_{3} \approx 1.202056903, \zeta_{4}=\pi^{4} / 90$, $\zeta_{5} \approx 1.036927755$. In this paper, all color factors are given for $N_{\mathrm{c}}=3$. Comparing the beta expressions here with those in Ref. [12], one would find a difference by a factor of $4^{i+1}$ due to the form of Eq. (1).

Previously, to solve Eq. (1), one first replaces the infinity there with a finite order, say 3 , and then expand the coupling to a series and seek the corresponding expansion coefficients. Here the problem is treated in a different way [8].

Summarizing the derivations in Ref. [8], we give the general solution of the RG equation Eq. (1) of the strong coupling as

$$
\alpha=\frac{L}{\beta_{0}} \sum_{i=0}^{\infty} \sum_{j=0}^{i} f_{i, j}\left(\frac{\beta_{1} L}{\beta_{0}^{2}}\right)^{i} \ln ^{j} L
$$

where $L=1 / \ln \left(u^{2} / \Lambda^{2}\right)$. The expansion coefficients $f_{i, j}$ are

$$
f_{i, j}=\frac{1}{(i-j) !} \sum_{l=0}^{i-j} f_{i-j, l} \sum_{s=0}^{j} \frac{(i-s) !}{(j-s) !} \bigsqcup_{1}^{l} \frac{1}{s}
$$

For the meaning of $\bigsqcup_{1}^{l} \frac{1}{s}$, see Eqs. (A8)-(A11). Because $\bigsqcup_{1}^{l} \frac{1}{s}=0$ when $s<l$, the lower limit of the summation index $s$ can be restricted to $s=l$, and the upper limit of the summation index $l$ can be constrained to $\min (i-j, j)$. For explicit results to order 6, please see in Ref. [8].

\section{FINITE-FORM EXPRESSIONS OF THE RUNNING COUPLING}

Generally, $f_{i, j}$ involves $\beta_{k}$ 's until $k \leq i$. In reality, we can only know the beta function to a finite order, say, $N$. Therefore, in perturbation theory we can only have an approximation of the exact solution. The question is now how to truncate the series so that it has the fastest convergent speed. In this section, we first discuss the two conventional approaches, and then develop another expansion which converges much faster.

\section{A. Standard truncation}

The conventional approach corresponds to simply replacing the $\infty$ in Eq. (3) with $N-1$, giving

$\alpha_{N}^{\mathrm{cv}}(u)=\frac{1}{\beta_{0} \ln \frac{u^{2}}{\Lambda^{2}}} \sum_{i=0}^{N-1}\left(\frac{\beta_{1}}{\beta_{0}^{2} \ln \frac{u^{2}}{\Lambda^{2}}}\right)^{i} \sum_{j=0}^{i}(-1)^{j} f_{i, j} \ln ^{j} \ln \frac{u^{2}}{\Lambda^{2}}$,

where the subscript cv indicates that it is the conventional standard expression. One can write it to arbitrary order according to the coefficients in the previous section. To order 5, for example, we explicitly write

$$
\begin{aligned}
\frac{\beta_{0}}{L} \alpha_{5}^{\mathrm{cv}}= & +\frac{\beta_{1} L}{\beta_{0}^{2}} \ln L+\frac{\beta_{1}^{2} L^{2}}{\beta_{0}^{4}}\left(U_{2}+\frac{\beta_{0} \beta_{2}}{\beta_{1}^{2}}\right) \\
& +\frac{\beta_{1}^{3} L^{3}}{\beta_{0}^{6}}\left[U_{3}+\left(3 \frac{\beta_{0} \beta_{2}}{\beta_{1}^{2}}-2\right) \ln L+\frac{\beta_{0}^{2} \beta_{3}}{2 \beta_{1}^{3}}\right] \\
& +\frac{\beta_{1}^{4} L^{4}}{\beta_{0}^{8}}\left[U_{4}+\left(6 \frac{\beta_{0} \beta_{2}}{\beta_{1}^{2}}-\frac{3}{2}\right) \ln ^{2} L\right. \\
& +\left(2 \frac{\beta_{0}^{2} \beta_{3}}{\beta_{1}^{3}}+3 \frac{\beta_{0} \beta_{2}}{\beta_{1}^{2}}-4\right) \ln L \\
& \left.-3 \frac{\beta_{0} \beta_{2}}{\beta_{1}^{2}}-\frac{\beta_{0}^{2} \beta_{3}}{6 \beta_{1}^{3}}+\frac{\beta_{0}^{2}}{3 \beta_{1}^{4}}\left(5 \beta_{2}^{2}+\beta_{0} \beta_{4}\right)\right] .
\end{aligned}
$$

where $U_{2}=\ln ^{2} L+\ln L-1, U_{3}=\ln ^{3} L+(5 / 2) \ln ^{2} L-$ $1 / 2, U_{4}=\ln ^{4} L+(13 / 3) \ln ^{3} L+7 / 6$. Because $\beta_{4}$ is not available presently, we can exactly calculate $\alpha_{N}^{\mathrm{cv}}(u)$, at most, to $N=4$ :

$$
\begin{gathered}
\alpha_{4}^{\mathrm{cv}}(u)=\frac{1}{\beta_{0} \ln \left(u^{2} / \Lambda^{2}\right)}-\frac{\beta_{1} \ln \ln \left(u^{2} / \Lambda^{2}\right)}{\beta_{0}^{3} \ln ^{2}\left(u^{2} / \Lambda^{2}\right)} \\
+\frac{\beta_{1}^{2}}{\beta_{0}^{5} \ln ^{3}\left(u^{2} / \Lambda^{2}\right)}\left[\left(\ln \ln \frac{u^{2}}{\Lambda^{2}}-\frac{1}{2}\right)^{2}+\frac{\beta_{0} \beta_{2}}{\beta_{1}^{2}}-\frac{5}{4}\right] \\
-\frac{\beta_{1}^{3}}{\beta_{0}^{7} \ln ^{4}\left(u^{2} / \Lambda^{2}\right)}\left[\left(\ln \ln \frac{u^{2}}{\Lambda^{2}}-\frac{5}{6}\right)^{3}-\frac{\beta_{0}^{2} \beta_{3}}{2 \beta_{1}^{3}}+\frac{233}{216}\right. \\
\left.+\left(3 \frac{\beta_{0} \beta_{2}}{\beta_{1}^{2}}-\frac{49}{12}\right) \ln \ln \frac{u^{2}}{\Lambda^{2}}\right] .
\end{gathered}
$$

Here the terms are grouped into four parts: two in the first line, one in the second line, and another one in the last two lines. The first one is $\alpha_{1}^{\mathrm{cv}}$, the first two give $\alpha_{2}^{\mathrm{cv}}$, and the first three are nothing but $\alpha_{3}^{\mathrm{cv}}$.

\section{B. Truncation in the RG equation}

Another way to obtain an approximation for the coupling is to truncate the series in Eq. (1) to a definite order, say $N$, i.e.

$$
\frac{\mathrm{d} \alpha}{\mathrm{d} \ln u^{2}}=-\sum_{i=0}^{N-1} \beta_{i} \alpha^{i+2}
$$


and then solve the corresponding differential equation exactly. In this case, we still have the exact series solution in Eq. (3), but all the $\beta_{i>N}$ should be set to zero. In the following, we discuss the compact form of the exact solution of Eq. (8).

If $N=1$, the solution is easy to get:

$$
\alpha_{1}^{\mathrm{rt}}=\frac{1}{\beta_{0} \ln \left(u^{2} / \Lambda^{2}\right)},
$$

which is the same as Eq. (5) at $N=1$.

If $N \geq 2$, we define

$$
W_{N}(\alpha) \equiv \beta_{0}^{2} \int_{0}^{\alpha}\left[\frac{1}{\beta_{0} x^{2}}-\frac{\beta_{1}}{\beta_{0}^{2} x}-\frac{1}{\sum_{i=0}^{N-1} \beta_{i} x^{i+2}}\right] \mathrm{d} x
$$

or, equivalently,

$$
W_{N}(\alpha) \equiv \int_{0}^{\alpha} \frac{\sum_{i=0}^{N-2}\left(\beta_{0} \beta_{i+2}-\beta_{1} \beta_{i+1}\right) \alpha^{i}-\beta_{0} \beta_{N} \alpha^{N-2}}{\sum_{j=0}^{N-1} \beta_{j} \alpha^{j}} \mathrm{~d} x
$$

then Eq. (8) can be integrated out to give

$$
\ln \frac{u^{2}}{\Lambda^{2}}=C^{\prime}+\frac{1}{\beta_{0} \alpha}+\frac{\beta_{1}}{\beta_{0}^{2}} \ln \alpha+\frac{1}{\beta_{0}^{2}} W_{N}(\alpha)
$$

To be consistent with the series solution, the constant of integration should be $C^{\prime}=\frac{\beta_{1}}{\beta_{0}^{2}}\left(\ln \beta_{0}+C\right)$. From Eq. (12), $u$ can be easily expressed as an explicit function of $\alpha$, i.e.,

$$
u^{2}(\alpha)=\Lambda^{2} \exp \left[\Theta_{N}(\alpha)+\frac{\beta_{1}}{\beta_{0}^{2}} C\right] .
$$

With the inverse function of $\Theta_{N}$, one then has an expression for the renormalization scale dependence of the coupling:

$$
\alpha_{N}^{\mathrm{rt}}=\Theta_{N}^{-1}\left[\ln \frac{u^{2}}{\Lambda^{2}}-\frac{\beta_{1}}{\beta_{0}^{2}} C\right]
$$

In Eqs. (13) and (14), the function $\Theta_{n}(\alpha)$ is defined as

$$
\Theta_{N}(\alpha) \equiv \frac{1}{\beta_{0} \alpha}+\frac{\beta_{1}}{\beta_{0}^{2}} \ln \left(\beta_{0} \alpha\right)+\frac{1}{\beta_{0}^{2}} W_{N}(\alpha) .
$$

The functions $W_{N}(\alpha)$ are

$$
\begin{aligned}
& W_{2}(\alpha)=-\beta_{1} \ln \left(1+\frac{\beta_{1}}{\beta_{0}} \alpha\right), \\
W_{3}(\alpha)= & \frac{2 \beta_{0} \beta_{2}-\beta_{1}^{2}}{\sqrt{4 \beta_{0} \beta_{2}-\beta_{1}^{2}}} \arctan \frac{\beta_{1}+2 \beta_{2} \alpha}{\sqrt{4 \beta_{0} \beta_{2}-\beta_{1}^{2}}} \\
& -\frac{2 \beta_{0} \beta_{2}-\beta_{1}^{2}}{\sqrt{4 \beta_{0} \beta_{2}-\beta_{1}^{2}}} \arctan \frac{\beta_{1}}{\sqrt{4 \beta_{0} \beta_{2}-\beta_{1}^{2}}} \\
& -\frac{\beta_{1}}{2} \ln \sum_{i=0}^{2} \frac{\beta_{i}}{\beta_{0}} \alpha^{i},
\end{aligned}
$$

and

$$
W_{4}(\alpha)=\sum_{i=0, \pm 1} \eta_{i} \ln \left(1+\frac{\alpha}{\alpha_{i}}\right)
$$

where $\alpha_{i}(i=0, \pm 1)$ are the three complex roots of the polynomial equation

$$
\beta_{0}-\beta_{1} \alpha+\beta_{2} \alpha^{2}-\beta_{3} \alpha^{3}=0,
$$

and $\eta_{i}$ is given by

$$
\eta_{i}=\frac{\beta_{0} \beta_{2}-\beta_{1}^{2}-\left(\beta_{0} \beta_{3}-\beta_{1} \beta_{2}\right) \alpha_{i}-\beta_{1} \beta_{3} \alpha_{i}^{2}}{\beta_{1}-2 \beta_{2} \alpha_{i}+3 \beta_{3} \alpha_{i}^{2}} .
$$

Please note, for the matching-invariant beta coefficients, which will be given in Eq. (C20), Eq. (19) has one real root and two conjugate complex roots. The real root is

$$
\alpha_{0}=\left(t_{1}+\sqrt{t_{1}^{2}+t_{2}^{3}}\right)^{1 / 3}-\frac{t_{2}}{\left(t_{1}+\sqrt{t_{1}^{2}+t_{2}^{3}}\right)^{1 / 3}}+\frac{\beta_{2}}{3 \beta_{3}} .
$$

with $t_{1}=(1 / 2) \beta_{0} / \beta_{3}-(1 / 6) \beta_{1} \beta_{2} / \beta_{3}^{2}+(1 / 27) \beta_{2}^{3} / \beta_{3}^{3}$, and $t_{2}=(1 / 3) \beta_{1} / \beta_{3}-(1 / 9) \beta_{2}^{2} / \beta_{3}^{2}$.

The expressions in Eqs. (9), (16), and (17) are all real numbers. The weak point of Eq. (18) involves calculations in the field of complex numbers. But the result is a real number for $W_{4}(\alpha)$. One can also give an expression to $W_{4}(\alpha)$ which concerns only real numbers. But the result is a little more complicated:

$$
\begin{aligned}
W_{4}(\alpha)= & \iota_{4}\left[\arctan \frac{\alpha-\iota_{1}}{\sqrt{\iota_{0}-\iota_{1}^{2}}}+\arctan \frac{\iota_{1}}{\sqrt{\iota_{0}-\iota_{1}^{2}}}\right] \\
& +\ln \frac{\left(1-2 \iota_{1} \alpha / \iota_{0}+\alpha^{2} / \iota_{0}\right)^{\iota_{3}}}{\left(1+\alpha / \alpha_{0}\right)^{\iota_{2}}},
\end{aligned}
$$

where

$$
\begin{aligned}
& \iota_{0}=\frac{\beta_{0}}{\beta_{3} \alpha_{0}}, \quad \iota_{1}=\frac{\beta_{0}-\beta_{1} \alpha_{0}}{2 \beta_{3} \alpha_{0}^{2}}=\frac{1}{2}\left(\alpha_{0}-\frac{\beta_{2}}{\beta_{3}}\right) \\
& \iota_{2}=\frac{\beta_{0}^{2}}{\alpha_{0}\left(3 \beta_{0}-2 \beta_{1} \alpha_{0}+\beta_{2} \alpha_{0}^{2}\right)}, \iota_{3}=\frac{1}{2}\left(\iota_{2}-\beta_{1}\right) \\
& \iota_{4}=\frac{\iota_{0}\left(\iota_{2} / \alpha_{0}+\beta_{2}-\beta_{1}^{2} / \beta_{0}\right)+\iota_{1}\left(\iota_{2}-\beta_{1}\right)}{\sqrt{\iota_{0}-\iota_{1}^{2}}} .
\end{aligned}
$$

To extend Eqs. (14) and (15) to $N=1$, one can let

$$
W_{1}=-\beta_{1} \ln \left(\beta_{0} \alpha\right) \text {. }
$$

When we have $W_{N}(\alpha)$, the coupling is then obtained by numerically solving the one-variable equation

$$
\frac{1}{\beta_{0} \alpha}+\frac{\beta_{1}}{\beta_{0}^{2}} \ln \left(\beta_{0} \alpha\right)+\frac{1}{\beta_{0}^{2}} W_{N}(\alpha)=\ln \frac{u^{2}}{\Lambda^{2}}-\frac{\beta_{1}}{\beta_{0}^{2}} C .
$$

For $N=2, \Theta_{N}^{-1}(\alpha)$ can also be expressed explicitly by using the physical branch of the Lambert function [14]. For higher $N$, however, it becomes difficult. 


\section{A new expression}

As an application of the general relation between $f_{i, j}$ and the beta coefficients, let's study another expansion which converges much faster. For this we rewrite the general series solution in Eq. (3) as

$$
\alpha=\frac{\beta_{0}}{\beta_{1}} \sum_{i=0}^{\infty} L^{* i+1} \sum_{j=0}^{i} f_{i, j} \ln ^{j} L \equiv \sum_{i=0}^{\infty} J_{i} .
$$

Representing the terms in this expansion with the corresponding coefficients $f_{i, j}$, all the terms can be arranged in a matrix as

$$
[\alpha]=\left[\begin{array}{ccccccc}
1 & 0 & 0 & 0 & 0 & 0 & \\
0 & 1 & 0 & 0 & 0 & 0 & \\
f_{2,0} & 1 & 1 & 0 & 0 & 0 & \\
f_{3,0} & f_{3,1} & 5 / 2 & 1 & 0 & 0 & \vdots \\
f_{4,0} & f_{4,1} & f_{4,2} & 13 / 3 & 1 & 0 & \\
f_{5,0} & f_{5,1} & f_{5,2} & f_{5,3} & 77 / 12 & 1 &
\end{array}\right] .
$$

The standard expansion corresponds to summing the terms row by row from the rho 0 to $N-1$. Then, obviously, an infinite number of terms, such as $f_{j, j}(j \geq N)$ on the diagonal and $f_{j+1, j}(j \geq N)$ on the next to diagonal etc, are lost, though these terms are all known as in Eq. (4). Generally, the terms $f_{j+k, j}$ on the $k$ th next to diagonal involves only $\beta_{i \leq k}$. But all the terms $f_{j+k, j}$ with $k<N$ and $j \geq N$ are known and lost, though no such terms are zero even if one sets all $\beta_{i>1}$ to zero.

To include the contribution from the terms just mentioned, Peng consider to sum over diagonals, which can be achieved by taking $i=j+k$ in Eq. (26), then, the following formula is obtained through mathematical derivation.

$$
\alpha=\frac{\chi_{0}}{\beta_{0}} \sum_{k=0}^{\infty} \sum_{l=0}^{k} f_{k, l} \chi^{k} \ln ^{l} \kappa
$$

where

$$
\begin{aligned}
\kappa & \equiv \frac{1}{1-L^{*} \ln L}=\left[1+\frac{\beta_{1} \ln \ln \left(u^{2} / \Lambda^{2}\right)}{\beta_{0}^{2} \ln \left(u^{2} / \Lambda^{2}\right)}\right]^{-1}, \\
\chi_{0} & \equiv L \kappa=\frac{\beta_{0}^{2}}{\beta_{0}^{2} \ln \left(u^{2} / \Lambda^{2}\right)+\beta_{1} \ln \ln \left(u^{2} / \Lambda^{2}\right)}, \\
\chi & \equiv \frac{\beta_{1}}{\beta_{0}^{2}} \chi_{0}=\frac{\beta_{1}}{\beta_{0}^{2} \ln \left(u^{2} / \Lambda^{2}\right)+\beta_{1} \ln \ln \left(u^{2} / \Lambda^{2}\right)} .
\end{aligned}
$$

Then, the new expression for the QCD running coupling can rewrite as

$$
\alpha_{N}^{\mathrm{fs}}=\frac{\beta_{0}}{\beta_{1}} \sum_{k=0}^{N-1}\left(\frac{\beta_{1}}{\beta_{0}^{2}} \kappa L\right)^{k+1} \sum_{l=0}^{k} f_{k, l} \ln ^{l} \kappa .
$$

To leading order, it is simply

$$
\alpha_{1}^{\mathrm{fs}}=\frac{\kappa}{\beta_{0} \ln \left(u^{2} / \Lambda^{2}\right)}=\frac{\beta_{0}}{\beta_{0}^{2} \ln \left(u^{2} / \Lambda^{2}\right)+\beta_{1} \ln \ln \left(u^{2} / \Lambda^{2}\right)} .
$$

To the next to leading order, it becomes

$$
\begin{aligned}
\alpha_{2}^{\mathrm{fs}}= & \frac{\beta_{0}}{\beta_{0}^{2} \ln \left(u^{2} / \Lambda^{2}\right)+\beta_{1} \ln \ln \left(u^{2} / \Lambda^{2}\right)} \\
& -\frac{\beta_{0} \beta_{1} \ln \left[1+\frac{\beta_{1} \ln \ln \left(u^{2} / \Lambda^{2}\right)}{\beta_{0}^{2} \ln \left(u^{2} / \Lambda^{2}\right)}\right]}{\left[\beta_{0}^{2} \ln \left(u^{2} / \Lambda^{2}\right)+\beta_{1} \ln \ln \left(u^{2} / \Lambda^{2}\right)\right]^{2}} \\
= & \frac{\kappa}{\beta_{0} \ln \left(u^{2} / \Lambda^{2}\right)}+\frac{\beta_{1} \kappa^{2} \ln \kappa}{\beta_{0}^{3} \ln ^{2}\left(u^{2} / \Lambda^{2}\right)} .
\end{aligned}
$$

To order 3 , it is

$$
\begin{aligned}
\alpha_{3}^{\mathrm{fs}}= & \frac{\kappa}{\beta_{0} \ln \left(u^{2} / \Lambda^{2}\right)}+\frac{\beta_{1} \kappa^{2} \ln \kappa}{\beta_{0}^{3} \ln ^{2}\left(u^{2} / \Lambda^{2}\right)} \\
& +\frac{\beta_{1}^{2} \kappa^{3}\left[\left(\ln \kappa+\frac{1}{2}\right)^{2}+\frac{\beta_{0} \beta_{2}}{\beta_{1}^{2}}-\frac{5}{4}\right]}{\beta_{0}^{5} \ln ^{3}\left(u^{2} / \Lambda^{2}\right)} .
\end{aligned}
$$

Presently we can give it to order 4 :

$$
\begin{aligned}
\alpha_{4}^{\mathrm{fs}}= & \frac{\beta_{0}}{\beta_{1}}\left[\chi+\chi^{2} \ln \kappa+\chi^{3}\left(\ln ^{2} \kappa+\ln \kappa+\frac{\beta_{0} \beta_{2}}{\beta_{1}^{2}}-1\right)\right. \\
& \left.\chi^{4}\left(\ln ^{3} \kappa+\frac{5}{2} \ln ^{2} \kappa+f_{3,1} \ln \kappa+f_{3,0}\right)\right],
\end{aligned}
$$

where $f_{3,0}$ and $f_{3,1}$ are given in Ref. [8], $\kappa$ is in Eq. (29), and $\chi$ in Eq. (31). For convenience, they can be listed here: $f_{3,0}=\left(\beta_{0}^{2} \beta_{3} / \beta_{1}^{3}-1\right) / 2, f_{3,1}=3 \beta_{0} \beta_{2} / \beta_{1}^{2}-2$, $\kappa=\left[1+\left(\beta_{1} / \beta_{0}^{2}\right) \ln \ln \left(u^{2} / \Lambda^{2}\right) / \ln \left(u^{2} / \Lambda^{2}\right)\right]^{-1}$, and $\chi=$ $\beta_{1} /\left[\beta_{0}^{2} \ln \left(u^{2} / \Lambda^{2}\right)+\beta_{1} \ln \ln \left(u^{2} / \Lambda^{2}\right)\right]$.

\section{Comparison of the three expressions}

Now we have three expressions for the coupling, i.e., Eqs. (32), (14), and (5). These expressions can be generally represented by

$$
\alpha=\alpha_{N}^{(i)}\left(u, N_{\mathrm{f}}, \Lambda, C\right),
$$

where $i=\mathrm{cv}$, rt, and fs represent, respectively, the expressions in Eqs. (5), (14), and (32). Therefore, the coupling depends not merely on the renormalization point $u$, but also on $i, N, N_{\mathrm{f}}$, i.e., for a theoretical value of the coupling, one must know it was calculated with which expression, to how high order, and for how many number of flavors.

In Eq. (37), there are other two arbitrary constants $\Lambda$ and $C$. Because the RG equation (1) or (C17) is of the first order, only one of them is independent. So we can arbitrarily take one of them, while the other one is determined by giving an initial condition. Although the freedom $C$ can be useful, e.g., for an optimization of the finite-order perturbation calculations and construction of universal $N_{\mathrm{f}}$-independent $\Lambda$ [16] etc, it nearly becomes standard, nowadays, to take $C=0[7,13]$, which makes 
the expression simpler (one would fail in Eq. (9) to determine $C$ for a given $\Lambda$ value because Eq. (9) has nothing to do with $C$ ), and [17]

$$
\alpha_{N}^{(i)}\left(M_{\mathrm{Z}}, N_{\mathrm{f}}, \Lambda, 0\right)=0.1179 / \pi,
$$

where $M_{\mathrm{Z}}=91.1876 \mathrm{GeV}$ is the mass of $\mathrm{Z}$ bosons. With the initial condition in Eq. (38), we can determine the scale $\Lambda$.

Please note, the experimental average of $\alpha_{s}\left(M_{\mathrm{Z}}\right)$ has moved by less than $1 \sigma$ from the previous one in Ref. [4].

From Eq. (38) we know that the scale $\Lambda$ depends on $N_{\mathrm{f}}, N$, i, i.e.,

$$
\Lambda=\Lambda_{n}(N, i)
$$

Here $n=N_{\mathrm{f}}=3,4,5,6$, i.e., setting $C=0$ requires distinct $\Lambda$ for different effective flavor regimes, and we use $\Lambda_{3}, \Lambda_{4}, \Lambda_{5}$, and $\Lambda_{6}$ for $m_{s}<u<m_{c}, m_{c}<u<$ $m_{b}, m_{b}<u<m_{t}$, and $u>m_{t}$, respectively, where the relevant quark thresholds are taken, in the present calculations, to be $m_{t}=172.76 \mathrm{GeV}, m_{b}=4.18 \mathrm{GeV}$, $m_{c}=1.27 \mathrm{GeV}$, and $m_{s}=93 \mathrm{MeV}[17]$.

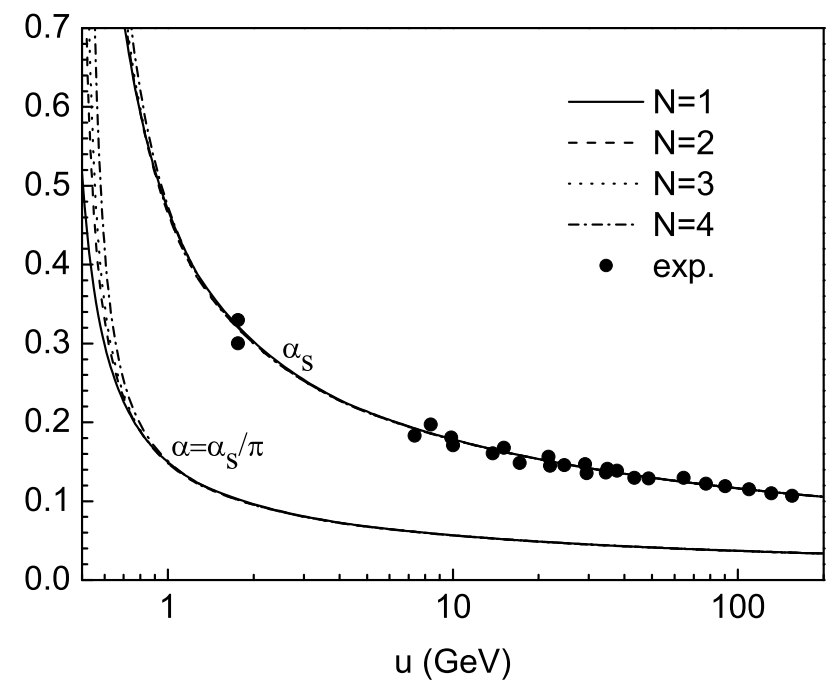

FIG. 1. The QCD coupling as a function of the 't Hooft unit of mass $u$, calculated by the new expression in Eq. (28) for the order $N$ from 1 to 4 . in Tab. I. The full dots are the experimental data of, in increasing $u, \tau$ decay, low $\mathrm{Q}^{2}$ cont, and EW precision fit from the $\mathrm{N}^{3} \mathrm{LO}$ data, $\mathrm{e}^{+} \mathrm{e}^{-}$and $\mathrm{pp} / \mathrm{p} \overline{\mathrm{p}}$ event shapes from the NNLO data, Heavy Quarkonia, DIS jets, and pp event shape from the NLO data.

In Fig. 1, the coupling is shown as a function of the renormalization point, calculated from Eq. (32) with the order $N$ from 1 to 4 . Please note, the beta coefficients used in the calculation are, in fact, the primed beta coefficients in Eq. (C20), rather than the original ones in Eq. (2). Therefore, the corresponding coupling is mathchinginvariant, to be discussed in detail a little later. The same calculation has also been performed from the conventional expansion in Eq. (5). The relative difference

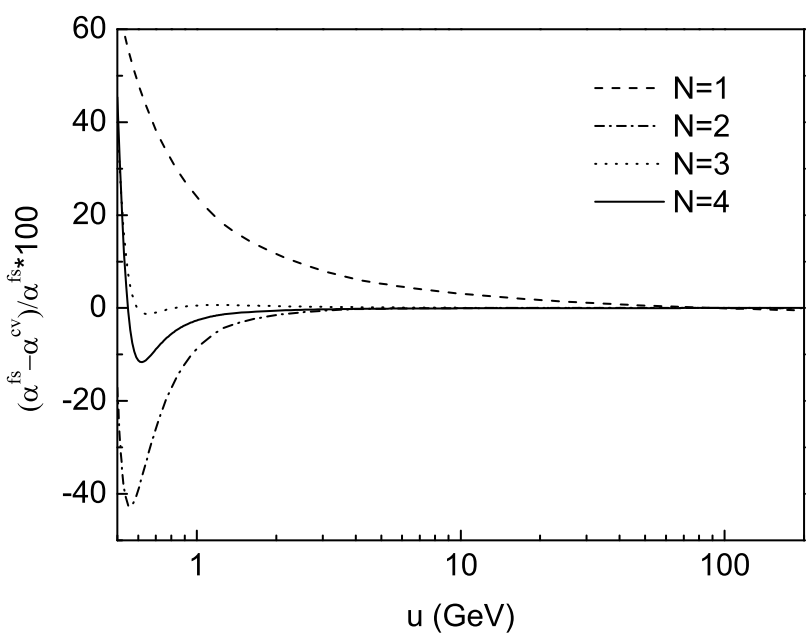

FIG. 2. The relative difference between the new expansion in Eq. (28) and the conventional expansion in Eq. (26).

TABLE I. The QCD scale parameter $\Lambda$ for the order $N$ from 1 to 4 and the number of flavors from 3 to 6 . There are three rows for each $\Lambda_{n}$ : the first row is calculated from the new expression in Eq. (32), the second row from the conventional standard expansion in Eq. (5), and the third row from Eq. (14).

\begin{tabular}{ccccc}
\hline$\Lambda(\mathrm{MeV})$ & $N=1$ & $N=2$ & $N=3$ & $N=4$ \\
\hline \multirow{3}{*}{$\Lambda_{3}$} & 314.987 & 336.766 & 330.620 & 329.181 \\
& 141.792 & 367.144 & 324.305 & 327.787 \\
& 141.788 & 355.335 & 328.907 & 329.829 \\
\hline \multirow{3}{*}{$\Lambda_{4}$} & 275.009 & 291.038 & 288.796 & 288.129 \\
& 118.981 & 321.668 & 285.921 & 287.621 \\
& 118.983 & 300.259 & 285.120 & 288.258 \\
\hline \multirow{3}{*}{$\Lambda_{5}$} & 199.080 & 207.159 & 207.732 & 207.450 \\
& 87.3112 & 224.966 & 207.199 & 207.199 \\
& 87.3112 & 210.699 & 204.334 & 207.464 \\
\hline \multirow{2}{*}{$\Lambda_{6}$} & 84.2465 & 86.4323 & 87.4815 & 87.3847 \\
& 42.3769 & 90.9752 & 87.6606 & 87.2738 \\
& 42.3860 & 87.3419 & 85.8268 & 87.4247 \\
\hline
\end{tabular}

between the results from Eq. (32) and Eq. (5) is shown in Fig. 2. It can be seen that, with decreasing $u$, the difference becomes more and more significant.

To compare convergence speed of three expressions in Eqs. (32), (5), and (14), all the $\Lambda_{n}(N, i)$, where $n=3$ $6, N=1-4$, and $i=\mathrm{cv} / \mathrm{rt} / \mathrm{fs}$, are listed in Tab. I. There are three rows corresponding to each $\Lambda_{n}$ : the first row is for Eq. (32), the second row for Eq. (5), and the third row for Eq. (14). It is obvious that the new expression in Eq. (32) converges much faster than the previous ones. Even at the leading-order $(N=1)$, the corresponding $\Lambda_{n}$ from Eq. (32) has already reached less than $5 \%$ close to the value at order 4 , while the other two have still been $50 \%$ away from the order- 4 value. The rapid convergent speed of the new expression in Eq. (32) can also be directly seen from Fig. 3(a), where the leading order coupling from the new expression (dashed line) is compared with the one from the previous expression (dotted line) and the order- 

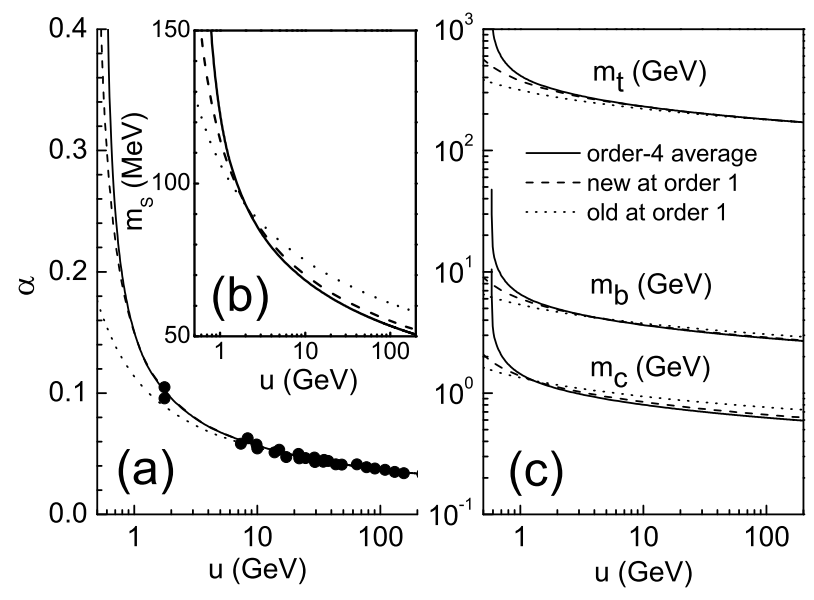

FIG. 3. QCD running coupling and quark masses in different expressions. The full line is the average value of the new and previous approaches at order 4 , the dashed and dotted lines are, respectively, from the new expressions in Eq. (32) and previous expressions in Eq. (14), at leading order. The new expression converges much faster.

4 average of the three expressions (solid line).

It is easy to understand the faster convergence of $\alpha^{\text {fs }}$ than $\alpha^{\mathrm{cv}}$ due to the inclusion of an infinite number of known logarithmic terms in a closed form. To understand that $\alpha^{\mathrm{fs}}$ converges faster than $\alpha^{\mathrm{rt}}$, we can expand $\alpha_{N}^{\mathrm{rt}}$ to a series which is nothing but exactly the same as that in Eq. (3) with all $\beta_{i \geq N}$ set to zero. Therefore, $\alpha^{\text {rt }}$ contains more terms than $\alpha^{\mathrm{cv}}$ and $\alpha^{\mathrm{fs}}$. However, the extra terms, which are included in $\alpha^{\mathrm{rt}}$ but not in $\alpha^{\mathrm{fs}}$, are incorrect because all $\beta_{i \geq N}$ have been set to zero.

\section{MATCHING-INVARIANT QUARK MASSES}

The renormalization equation for the quark masses is

$$
\frac{\mathrm{d} \ln m_{\mathrm{q}}}{\mathrm{d} \ln u^{2}}=-\sum_{i=0}^{\infty} \gamma_{i} \alpha^{i+1} \equiv \gamma(\alpha) .
$$

where the quark mass anomalous dimension $\gamma_{i}$ is known to 4-loop level [22, 23]:

$$
\begin{aligned}
\gamma_{0}= & 1, \quad \gamma_{1}=101 / 24-(5 / 36) N_{\mathrm{f}} \\
\gamma_{2}= & \frac{1249}{64}-\left(\frac{5}{6} \zeta_{3}+\frac{277}{216}\right) N_{\mathrm{f}}-\frac{35}{1296} N_{\mathrm{f}}^{2} \\
\gamma_{3}= & \frac{4603055}{41472}+\frac{530}{27} \zeta_{3}-\frac{275}{8} \zeta_{5} \\
& -\left(\frac{91723}{6912}+\frac{2137}{144} \zeta_{3}-\frac{575}{72} \zeta_{5}-\frac{11}{288} \pi^{4}\right) N_{\mathrm{f}} \\
& +\left(\frac{2621}{31104}+\frac{25}{72} \zeta_{3}-\frac{\pi^{4}}{432}\right) N_{\mathrm{f}}^{2} \\
& +\left(\frac{\zeta_{3}}{108}-\frac{83}{15552}\right) N_{\mathrm{f}}^{3} .
\end{aligned}
$$

Suppose the matching condition for quark masses is

$$
m_{q}^{*}=m_{q} \sum_{i=0}^{\infty} \sum_{j=0}^{i} D_{i, j} \alpha^{i} \ln ^{j} \frac{m_{\mathrm{h}}^{2}}{u^{2}},
$$

i.e., $m_{q}$ is the quark mass in the full $N_{\mathrm{f}}$-flavor theory for flavor $q$, while $m_{q}^{*}$ belongs to the corresponding effective theory with $N_{\mathrm{f}}-1$ flavors. $m_{q}^{*}$ should also satisfy $\mathrm{RG}$ equation, and thus satisfy

$$
\frac{\mathrm{d} m_{q}^{*}}{m_{q} \mathrm{~d} \ln u^{2}}=-\frac{m_{q}^{*}}{m_{q}} \sum_{k=0}^{\infty} \gamma_{k}^{*} \alpha^{* k+2} .
$$

Substituting Eq. (42) into Eq, (43), and then comparing coefficients will give $D_{i, j>0}$ recursively

$$
\begin{aligned}
D_{i, j}= & \frac{1}{j} \sum_{i^{\prime}=0}^{\bar{\imath}} \sum_{j^{\prime}=J^{*}}^{\bar{\jmath}} D_{\bar{\imath}-i^{\prime}, \bar{\jmath}-j^{\prime}} \sum_{k=0}^{i^{\prime}-j^{\prime}} \gamma_{k}^{*} \bigsqcup_{0,0}^{k+1} C_{i^{\prime}-k, j^{\prime}} \\
& -\frac{1}{j} \sum_{k=\bar{\jmath}}^{\bar{i}}\left(\gamma_{k-\bar{\jmath}} D_{\bar{\imath}+\bar{\jmath}-k, \bar{\jmath}}+k \beta_{\bar{\imath}-k} D_{k, \bar{\jmath}}\right) \\
& -2 \sum_{k=j}^{i-1} \gamma_{i-1-k} D_{k, j},
\end{aligned}
$$

where $J^{*}=\max \left(0, j-i+i^{\prime}\right), \bar{\imath}=i-1, \bar{\jmath}=j-1$.

To solve $D_{i, j>0}$ from Eq. (44), we should know $D_{i, 0}$. Here are the results for $D_{i, 0}$ to three-loop level [18, 19]:

$$
\begin{aligned}
& D_{0,0}=1, \quad D_{1,0}=0, \quad D_{2,0}=89 / 432, \\
& D_{3,0}=\left(\frac{1327}{11664}-\frac{2}{27} \zeta_{3}\right) N_{\mathrm{f}}+D_{3,0}^{(0)} .
\end{aligned}
$$

With the quadrilogarithm $\operatorname{Li}_{4}(1 / 2)=\operatorname{polylog}(4,1 / 2)=$ $\sum_{k=1}^{\infty}(1 / 2)^{k} / k^{4} \approx 0.5174790617$, the constant $D_{3,0}^{(0)}$ is $D_{3,0}^{(0)} \equiv 10477 / 11664-(343 / 864) \zeta_{3}+\frac{103}{6480} \pi^{4}-\frac{4}{9} \operatorname{Li}_{4}(1 / 2)+$ $\frac{\ln ^{2} 2}{54}\left(\pi^{2}-\ln ^{2} 2\right) \approx 1.822899133$ [24].

Solving Eq. (44) with the aid of Eq. (45) gives

$$
\begin{aligned}
D_{1,1} & =0, \quad D_{2,1}=5 / 18, \quad D_{2,2}=1 / 3, \\
D_{3,1} & =D_{3,1}^{(0)}+\frac{53}{216} N_{\mathrm{f}}, D_{3,2}=\frac{175}{108}, \\
D_{3,3} & =\frac{1}{27}\left(2 N_{\mathrm{f}}-31\right), D_{4,1}=D_{4,1}^{(0)}+D_{4,1}^{(1)} N_{\mathrm{f}}+D_{4,1}^{(2)} N_{\mathrm{f}}^{2}, \\
D_{4,2} & =D_{4,2}^{(0)}-\frac{8321}{2592} N_{\mathrm{f}}+\frac{31}{324} N_{\mathrm{f}}^{2}, D_{4,3}=\frac{23}{36} N_{\mathrm{f}}-\frac{2615}{324}, \\
D_{4,4} & =\frac{1039}{216}-\frac{16}{27} N_{\mathrm{f}}+\frac{N_{\mathrm{f}}^{2}}{54}, \cdots
\end{aligned}
$$

where $D_{3,1}^{(0)}=(5 / 3) \zeta_{3}-7 / 1296 \approx 1.998026937, D_{4,1}^{(0)}=$ $644891 / 20736-(33 / 2) D_{3,0}^{(0)}-35 \pi^{4} / 432+97175 \zeta_{3} / 4608-$ $575 \zeta_{5} / 36 \approx 1.91761725, D_{4,1}^{(1)}=D_{3,0}^{(0)}+\pi^{4} / 108-\zeta_{3} / 9-$ $35173 / 15552 \approx 0.3296349104, D_{4,1}^{(2)}=(3401 / 432-$ $\left.7 \zeta_{3}\right) / 54 \approx-0.0100317247, \quad D_{4,2}^{(0)}=14809 / 648-$ $155 \zeta_{3} / 12 \approx 7.32682673$. 
If the $m_{\mathrm{h}}$ in Eq. (42) is RG-invariant, the last term on the right hand side of Eq. (44) disappear. On the other hand, if one takes $u=m_{\mathrm{h}}$, then the matching condition Eqs. (42) becomes

$$
m_{q}^{*}=m_{q} \sum_{i=0}^{\infty} D_{i} \alpha^{i}
$$

where $D_{i} \equiv D_{i, 0}$. The matching condition for the running coupling is

$$
\alpha^{*}=\sum_{k=0}^{\infty} C_{k} \alpha^{k+1}
$$

where $C_{k} \equiv C_{k, 0}$ is written in the appendix B.

Suppose the matching-invariant quark masses are given by

$$
m_{q}^{\prime}=m_{q} \sum_{j=0}^{\infty} b_{j} \alpha^{j}
$$

then the matching condition for $m_{q}^{\prime}$ is

$$
m_{q}^{\prime *}=m_{q}^{*} \sum_{j=0}^{\infty} b_{j}^{*} \alpha^{* j},
$$

where $b_{i}$ depends on $N_{\mathrm{f}}$ and is to be determined by the condition $m_{\mathrm{q}}^{\prime *}=m_{q}^{\prime}$. Again a superscript star means decreasing $N_{\mathrm{f}}$ by one.

Substituting Eqs. (47) and (48) into Eq. (50), and then comparing the coefficients of $\alpha^{i}$ in the equality $m_{\mathrm{q}}^{\prime *}=$ $m_{q}^{\prime}$, we obtain

$$
b_{i}=\sum_{k=0}^{i} D_{i-k} \sum_{j=0}^{k} b_{j}^{*} \bigsqcup_{0}^{j} C_{k-j} .
$$

Express $b_{i}$ as a polynomial of $N_{\mathrm{f}}$, i.e.,

$$
b_{i}=\sum_{k=0}^{i} b_{i, k} N_{\mathrm{f}}^{i}
$$

then $b_{j}^{*}=\sum_{l=0}^{j} b_{j, l}\left(N_{\mathrm{f}}-1\right)^{l}$, and Eq. (51) becomes

$$
\sum_{k=0}^{i}\left[b_{i, k} N_{\mathrm{f}}^{k}-D_{i-k} \sum_{j=0}^{k} \sum_{l=0}^{j} b_{j, l}\left(N_{\mathrm{f}}-1\right)^{l} \bigsqcup_{0}^{j} C_{k-j}\right]=0
$$

whose solution is

$$
\begin{aligned}
b_{1,1}= & 0, \quad b_{2,1}=(89 / 432) b_{0,0}, \quad b_{2,2}=0, \\
b_{3,1}= & \left(\frac{7427}{7776}-\frac{125}{288} \zeta_{3}+\frac{\pi^{4}}{72}-\frac{B_{4}}{36}\right) b_{0,0} \\
& +\frac{155}{432} b_{1,0} \\
b_{3,2}= & \left(\frac{1327}{23328}-\frac{\zeta_{3}}{27}\right) b_{0,0}, \quad b_{3,3}=0,
\end{aligned}
$$

where $B_{4}=16 \operatorname{Li}_{4}(1 / 2)-13 \zeta_{4} / 2-4 \zeta_{2} \ln ^{2} 2+(2 / 3) \ln ^{4} 2 \approx$ -1.762800087 [24]. Taking the simplest nontrivial choice $b_{i, 0}=\delta_{i, 0}$ leads to

$$
b_{0}=1, \quad b_{1}=0, b_{2}=\frac{89}{432} N_{\mathrm{f}}, b_{3}=b_{3,1} N_{\mathrm{f}}+b_{3,2} N_{\mathrm{f}}^{2}, \cdots
$$

where

$$
\begin{aligned}
b_{3,1} & =\frac{7427}{7776}-\frac{125}{288} \zeta_{3}+\frac{\pi^{4}}{72}-\frac{B_{4}}{36} \\
& \approx 1.835262938 \\
b_{3,2} & =\frac{1327}{23328}-\frac{\zeta_{3}}{27} \approx 0.01236380468 .
\end{aligned}
$$

Explicitly, the matching-invariant quark mass is

$$
\frac{m_{q}^{\prime}}{m_{q}}=1+\frac{89}{432} N_{\mathrm{f}} \alpha^{2}+\left[b_{3,1}+b_{3,2} N_{\mathrm{f}}\right] N_{\mathrm{f}} \alpha^{3}+\cdots .
$$

The inverse relation of Eq. (49) is

$$
m_{q}=m_{q}^{\prime} \sum_{j=0}^{\infty} b_{j}^{\prime} \alpha^{\prime j}
$$

where the coefficients $b_{j}^{\prime}$ are linked to $b_{j}$ by solving

$$
\sum_{i=0}^{k} b_{k-i} \sum_{j=0}^{i} b_{j}^{\prime} \bigsqcup_{0}^{j} a_{i-j}=\delta_{k, 0}
$$

To three-loop level, one has the explicit expression

$$
\frac{m_{q}}{m_{q}^{\prime}}=1-\frac{89}{432} N_{\mathrm{f}} \alpha^{\prime 2}-\left[b_{3,1}+b_{3,2} N_{\mathrm{f}}\right] N_{\mathrm{f}} \alpha^{\prime 3}+\cdots
$$

where $b_{3,1}$ and $b_{3,2}$ are given in Eq. (56).

The RG equation for the new mass in the new coupling is

$$
\frac{\mathrm{d} \ln m_{q}^{\prime}}{\mathrm{d} \ln u^{2}}=-\sum_{i=0}^{\infty} \gamma_{i}^{\prime} \alpha^{\prime i+1}
$$

where $\gamma^{\prime}$ is the new gamma coefficients determined by

$$
\sum_{k=0}^{i} b_{i-k}\left[\sum_{j=0}^{k} \gamma_{j}^{\prime} \bigsqcup_{0}^{j+1} a_{k-j}-\gamma_{k}-(i-k) \beta_{k}\right]=0
$$

which is also a recursive relation. The solution is

$$
\begin{aligned}
& \gamma_{0}^{\prime}=\gamma_{0}, \gamma_{1}^{\prime}=\gamma_{1}, \gamma_{2}^{\prime}=\gamma_{2}-a_{2} \gamma_{0}+2 b_{2} \beta_{0}, \\
& \gamma_{3}^{\prime}=\gamma_{3}-2 a_{2} \gamma_{1}-a_{3} \gamma_{0}+2 b_{2} \beta_{1}+3 b_{3} \beta_{0}, \cdots
\end{aligned}
$$

Here $a_{0}=b_{0}=1$ and $a_{1}=b_{1}=0$ have been used. With the expressions for $a_{2}$ and $a_{3}$ in Eq. (C8), $b_{2}$ and $b_{3}$ in Eq. (55), the original beta and gamma coefficients in Eqs. (2) and (41), the primed gamma coefficients can be expressed as

$$
\gamma_{i}^{\prime}=\sum_{k=0}^{i} c_{i, k} N_{\mathrm{f}}^{k}
$$


with the color factors given by $c_{0,0}=1, c_{1,0}=$ $101 / 24, \quad c_{1,1}=-5 / 36, \quad c_{2,0}=1249 / 64, \quad c_{2,1}=$ $-29 / 96-5 \zeta_{3} / 6 \approx-1.303797419, \quad c_{2,2}=$ $79 / 1152, \gamma_{3,0}=4603055 / 41472+530 \zeta_{3} / 27-275 \zeta_{5} / 8 \approx$ $98.94341426, \gamma_{3,1}=-357941 / 41472-427261 \zeta_{3} / 27648+$ $575 \zeta_{5} / 72+11 \pi^{4} / 72-11 B_{4} / 48 \approx-3.640068635, \gamma_{3,2}=$ $-10291 / 62208+149 \zeta_{3} / 576-\pi^{4} / 108+B_{4} / 72 \approx$ $-0.7808994989, \quad \gamma_{3,3}=\zeta_{3} / 36-197 / 5832 \approx$ -0.0003886799877 .

\section{EXPRESSION FOR QUARK MASSES}

To solve Eq. (40), one can divide it by Eq. (1), giving

$$
\frac{d \ln m_{q}}{d \alpha}=\frac{\gamma(\alpha)}{\beta(\alpha)}=\frac{\gamma_{0}}{\beta_{0} \alpha}+\dot{\gamma}(\alpha)
$$

where

$$
\dot{\gamma}(\alpha) \equiv \frac{\gamma(\alpha)}{\beta(\alpha)}-\frac{\gamma_{0}}{\beta_{0} \alpha}=\frac{\sum_{i=0}^{\infty}\left(\gamma_{i+1}-\frac{\gamma_{0}}{\beta_{0}} \beta_{i+1}\right) \alpha^{i}}{\sum_{j=0}^{\infty} \beta_{j} \alpha^{j}} .
$$

It is easy to check that $\dot{\gamma}(\alpha)$ is analytic at $\alpha=0$, and can thus be expanded to a Taylor series, i.e.,

$$
\dot{\gamma}(\alpha)=\sum_{k=0}^{\infty} \dot{\gamma}_{k} \alpha^{k}
$$

where the Taylor coefficients can be obtained either from the normal mathematical formula

$$
\dot{\gamma}_{k}=\left.\frac{1}{k !} \frac{\mathrm{d}^{k}}{\mathrm{~d} \alpha^{k}} \dot{\gamma}(\alpha)\right|_{\alpha=0}
$$

or, simply, from the recursive relation

$$
\dot{\gamma}_{k}=\frac{\gamma_{k+1}}{\beta_{0}}-\frac{\gamma_{0}}{\beta_{0}^{2}} \beta_{k+1}-\frac{1}{\beta_{0}} \sum_{l=0}^{k-1} \beta_{k-l} \dot{\gamma}_{l} .
$$

The following are the first several concrete expressions:

$$
\begin{aligned}
\dot{\gamma}_{0}= & \gamma_{1} / \beta_{0}-\gamma_{0} \beta_{1} / \beta_{0}^{2}, \\
\dot{\gamma}_{1}= & \gamma_{2} / \beta_{0}-\left(\beta_{1} \gamma_{1}+\beta_{2} \gamma_{0}\right) / \beta_{0}^{2}+\beta_{1}^{2} \gamma_{0} / \beta_{0}^{3}, \\
\dot{\gamma}_{2}= & \gamma_{3} / \beta_{0}-\left(\beta_{1} \gamma_{2}+\beta_{2} \gamma_{1}+\beta_{3} \gamma_{0}\right) / \beta_{0}^{2} \\
& +\beta_{1}\left(\beta_{1} \gamma_{1}+2 \beta_{2} \gamma_{0}\right) / \beta_{0}^{3}-\beta_{1}^{3} \gamma_{0} / \beta_{0}^{4}, \\
& \ldots
\end{aligned}
$$

Integrating Eq. (65) leads to

$$
\ln \frac{m_{q}}{\hat{m}_{q}}=\frac{\gamma_{0}}{\beta_{0}} \ln \alpha+\int_{0}^{\alpha} \dot{\gamma}(x) \mathrm{d} x,
$$

where $\hat{m}_{q}$ is a RG-invariant dimensional parameter, or the constant of integration. Eq. (71) immediately gives

$$
m_{q}=\hat{m}_{q} \alpha^{\gamma_{0} / \beta_{0}} \exp \left[\int_{0}^{\alpha} \dot{\gamma}(x) \mathrm{d} x\right] \text {. }
$$

One can expand the last factor on the right hand side of Eq. (72) to a Taylor series, giving

$$
m_{q}=\hat{m}_{q} \alpha^{\gamma_{0} / \beta_{0}}\left(\sum_{i=0}^{\infty} \tilde{\gamma}_{i} \alpha^{i}\right)
$$

where

$$
\tilde{\gamma}_{i}=\left.\frac{1}{i !} \frac{d^{i}}{d \alpha^{i}} \exp \left[\int_{0}^{\alpha} \dot{\gamma}(x) \mathrm{d} x\right]\right|_{\alpha=0} .
$$

In fact, substituting Eq. (67) into Eq. (72), integrating term by term, aplying $e^{x}=\sum_{k=0}^{\infty} x^{k} / k$ !, and then using the square-cup operator, we can give a general expression

$$
\tilde{\gamma}_{i}=\sum_{k=0}^{i} \frac{1}{(i-k) !} \bigsqcup_{0}^{i-k} \frac{\dot{\gamma}_{k}}{k+1} .
$$

Explicitly, the tilde gamma can be listed as

$$
\begin{aligned}
\tilde{\gamma}_{0}= & 1, \tilde{\gamma}_{1}=\dot{\gamma}_{0}=\gamma_{1} / \beta_{0}-\gamma_{0} \beta_{1} / \beta_{0}^{2}, \\
\tilde{\gamma}_{2}= & \left(\dot{\gamma}_{0}^{2}+\dot{\gamma}_{1}\right) / 2 \\
= & \frac{1}{2}\left[\gamma_{2} / \beta_{0}+\left(\gamma_{1}^{2}-\gamma_{1} \beta_{1}-\gamma_{0} \beta_{2}\right) / \beta_{0}^{2}\right. \\
& \left.\quad-\gamma_{0} \beta_{1}\left(2 \gamma_{1}-\beta_{1}\right) / \beta_{0}^{3}+\gamma_{0}^{2} \beta_{1}^{2} / \beta_{0}^{4}\right] \\
\tilde{\gamma}_{3}= & \frac{1}{6} \dot{\gamma}_{0}^{3}+\frac{1}{2} \dot{\gamma}_{0} \dot{\gamma}_{1}+\frac{1}{3} \dot{\gamma}_{2} \\
= & \frac{\gamma_{3}}{3 \beta_{0}}+\frac{3 \gamma_{1} \gamma_{2}-2\left(\gamma_{0} \beta_{3}+\gamma_{1} \beta_{2}+\gamma_{2} \beta_{1}\right)}{6 \beta_{0}^{2}} \\
+\frac{\gamma_{1}^{3}}{+}+4 \gamma_{0} \beta_{1} \beta_{2}+2 \gamma_{1} \beta_{1}^{2}-3\left(\gamma_{1}^{2} \beta_{1}+\gamma_{0} \gamma_{2} \beta_{1}+\gamma_{0} \gamma_{1} \beta_{2}\right) & 6 \beta_{0}^{3} \\
& +\frac{\gamma_{0} \beta_{1}\left(3 \gamma_{0} \beta_{2}+6 \gamma_{1} \beta_{1}-2 \beta_{1}^{2}-3 \gamma_{1}^{2}\right)}{6 \beta_{0}^{4}} \\
& +\frac{\gamma_{0}^{2} \beta_{1}^{2}\left(\gamma_{1}-\beta_{1}\right)}{2 \beta_{0}^{5}}-\frac{\gamma_{0}^{3} \beta_{1}^{3}}{6 \beta_{0}^{6}},
\end{aligned}
$$

To have a finite-form expression for quark masses, one can truncate the series in Eq. (73) to a finite order, say $N$, to give

$$
m_{q}=\hat{m}_{q} \alpha^{\gamma_{0} / \beta_{0}}\left(\sum_{i=0}^{N-1} \tilde{\gamma}_{i} \alpha^{i}\right)
$$

Another approach is to truncate the series $\gamma(x)$ in Eq. (72). In this way the solution in Eq. (72) becomes

$$
m_{q}=\hat{m}_{q} \alpha^{\gamma_{0} / \beta_{0}} \exp \left[w_{N}(\alpha)\right] .
$$

where

$$
w_{N}(\alpha) \equiv \int_{0}^{\alpha} \frac{\sum_{j=0}^{N-2}\left(\gamma_{j+1}-\frac{\gamma_{0}}{\beta_{0}} \beta_{j+1}\right) x^{j}}{\sum_{i=0}^{N-1} \beta_{i} x^{i}} \mathrm{~d} x
$$


Explicitly, one can derive

$$
\begin{aligned}
& w_{1}(\alpha)=0, \\
& w_{2}(\alpha)=\left(\frac{\gamma_{1}}{\beta_{1}}-\frac{\gamma_{0}}{\beta_{0}}\right) \ln \left(1+\frac{\beta_{1}}{\beta_{0}} \alpha\right), \\
& w_{3}(\alpha)=\frac{1}{2}\left(\frac{\gamma_{2}}{\beta_{2}}-\frac{\gamma_{0}}{\beta_{0}}\right) \ln \left(1+\frac{\beta_{1}}{\beta_{0}} \alpha+\frac{\beta_{2}}{\beta_{0}} \alpha^{2}\right) \\
& -\frac{\left(\frac{\gamma_{0}}{\beta_{0}}-2 \frac{\gamma_{1}}{\beta_{1}}+\frac{\gamma_{2}}{\beta_{2}}\right)}{\sqrt{4 \beta_{0} \beta_{2} / \beta_{1}^{2}-1}}\left[\arctan \frac{1+2 \frac{\beta_{2}}{\beta_{1}} \alpha}{\sqrt{4 \beta_{0} \beta_{2} / \beta_{1}^{2}-1}}\right. \\
& \left.-\arctan \frac{1}{\sqrt{4 \beta_{0} \beta_{2} / \beta_{1}^{2}-1}}\right] \text {, } \\
& w_{4}(\alpha)=\iota_{4}\left[\arctan \frac{\alpha-\iota_{1}}{\sqrt{\iota_{0}-\iota_{1}^{2}}}+\arctan \frac{\iota_{1}}{\sqrt{\iota_{0}-\iota_{1}^{2}}}\right] \\
& +\ln \frac{\left(1-2 \iota_{1} \alpha / \iota_{0}+\alpha^{2} / \iota_{0}\right)^{\iota_{3}}}{\left(1+\alpha / \alpha_{0}\right)^{\iota_{2}}},
\end{aligned}
$$

where

$$
\begin{aligned}
\iota_{0} & =\frac{\beta_{0}}{\beta_{3} \alpha_{0}}, \iota_{1}=\frac{1}{2}\left(\alpha_{0}-\frac{\beta_{2}}{\beta_{3}}\right), \\
\iota_{2} & =\frac{\sum_{i=1}^{3}(-1)^{i}\left(\gamma_{i}-\frac{\gamma_{0}}{\beta_{0}} \beta_{i}\right) \alpha_{0}^{i-1}}{\beta_{2} \alpha_{0}-2 \beta_{1}+3 \beta_{0} / \alpha_{0}}, \\
\iota_{3} & =\frac{1}{2}\left(\iota_{2}+\frac{\gamma_{3}}{\beta_{3}}-\frac{\gamma_{0}}{\beta_{0}}\right), \\
\iota_{4} & =\frac{\left(\gamma_{1} / \beta_{3}-\gamma_{0} \beta_{1} / \beta_{0} \beta_{3}+\iota_{0} \iota_{2}\right) / \alpha_{0}+2 \iota_{1} \iota_{3}}{\sqrt{\iota_{0}-\iota_{1}^{2}}} .
\end{aligned}
$$

Previous calculations usually use Eq. (77) or (78) to calculate quark running masses. The weak point is obvious: the dependence on $u$ is not direct, but through $\alpha$ whose dependence on $u$ is not exactly known, which causes extra imprecision. To connect $m_{q}$ directly to $u$, we can substitute the general expression for $\alpha$ in Eq. (3) into Eq. (73). In fact from Eq. (3) we have

$$
\begin{aligned}
& \alpha^{\frac{\gamma_{0}}{\beta_{0}}}=\left(\frac{L}{\beta_{0}}\right)^{\gamma_{0} / \beta_{0}}\left[1+\sum_{i=1}^{\infty} \sum_{j=0}^{i} f_{i, j} L^{* i} \ln ^{j} L\right]^{\gamma_{0} / \beta_{0}} \\
& =\left(\frac{L}{\beta_{0}}\right)^{\frac{\gamma_{0}}{\beta_{0}}} \sum_{k=0}^{\infty} \frac{\prod_{l=0}^{k-1}\left(\frac{\gamma_{0}}{\beta_{0}}-l\right)}{k !}\left[\sum_{i=1}^{\infty} \sum_{j=0}^{i} f_{i, j} L^{* i} \ln ^{l} L\right]^{k} .
\end{aligned}
$$

Using the square-cup operator, this becomes

$$
\frac{\alpha^{\frac{\gamma_{0}}{\beta_{0}}}}{\left(\frac{L}{\beta_{0}}\right)^{\frac{\gamma_{0}}{\beta_{0}}}}=\sum_{i=0}^{\infty} \sum_{j=0}^{i}\left(\sum_{k=0}^{i} \frac{\prod_{l=0}^{k-1}\left(\frac{\gamma_{0}}{\beta_{0}}-l\right)}{k !} \bigsqcup_{1,0}^{k} f_{i, j}\right) L^{* i} \ln ^{j} L .
$$

Similarly, we get

$$
\sum_{i=0}^{\infty} \tilde{\gamma}_{i} \alpha^{i}=\sum_{i^{\prime}=0}^{\infty} \sum_{j^{\prime}=0}^{i^{\prime}}\left(\sum_{s=0}^{i^{\prime}-j^{\prime}} \grave{\gamma}_{s} \bigsqcup_{0,0}^{s} f_{i^{\prime}-s, j^{\prime}}\right) L^{* i^{\prime}} \ln ^{j^{\prime}} L,
$$

where the graved gamma function is given by

$$
\grave{\gamma}_{s} \equiv\left(\frac{\beta_{0}}{\beta_{1}}\right)^{s} \tilde{\gamma}_{s}
$$

Explicitly, the first several graved beta coefficients are

$$
\begin{aligned}
& \grave{\gamma}_{0}=1, \grave{\gamma}_{1}=\frac{\gamma_{1}}{\beta_{1}}-\frac{\gamma_{0}}{\beta_{0}}, \grave{\gamma}_{2}=\frac{\beta_{0}^{2}}{2 \beta_{1}^{2}}\left(\dot{\gamma}_{0}^{2}+\dot{\gamma}_{1}\right), \\
& \grave{\gamma}_{3}=\frac{\beta_{0}^{3}}{6 \beta_{1}^{3}}\left(\dot{\gamma}_{0}^{3}+3 \dot{\gamma}_{0} \dot{\gamma}_{1}+2 \dot{\gamma}_{2}\right), \cdots
\end{aligned}
$$

Substituting Eqs. (85) and (86) into Eq. (73) gives

$$
m_{q}=m_{q n} L^{\gamma_{0} / \beta_{0}} \sum_{i=0}^{\infty} \sum_{j=0}^{i} F_{i, j} L^{* i} \ln ^{j} L
$$

where the scale parameters for the quark mass $m_{q}$ has been naturally changed to

$$
m_{q n} \equiv \hat{m}_{q} \beta_{0}^{-\gamma_{0} / \beta_{0}} .
$$

Eq. (90) means that the quark mass is proportional to $L^{\gamma_{0} / \beta_{0}}$ at small $L$ or large $u$, and the proportion coefficient is nothing but $m_{q n}$.

The expansion coefficients $F_{i, j}$ in Eq. (90) are

$$
F_{i, j}=\sum_{i^{\prime}, j^{\prime}, k, s} \grave{\gamma}_{s} \frac{\prod_{l=0}^{k-1}\left(\gamma_{0} / \beta_{0}-l\right)}{k !} \bigsqcup_{1,0}^{k} f_{i-i^{\prime}, j-j^{\prime}} \bigsqcup_{0,0}^{s} f_{i^{\prime}-s, j^{\prime}}
$$

with the multi-summation being

$$
\sum_{i^{\prime}, j^{\prime}, k, s}=\sum_{i^{\prime}=0}^{i} \sum_{j^{\prime}=\max \left(0, j-i+i^{\prime}\right)}^{\min \left(j, i^{\prime}\right)} \sum_{k=0}^{i-i^{\prime}} \sum_{s=0}^{i^{\prime}-j^{\prime}}
$$

It is also easy to express $F_{i, j}$ as a linear combination of the graved gamma function, i.e.,

$$
\sum_{i^{\prime}, j^{\prime}, k, s}=\sum_{s=0}^{i-j} \sum_{k=0}^{i-s} \sum_{i^{\prime}=s}^{i-k} \sum_{j^{\prime}=\max \left(0, j-i+i^{\prime}\right)}^{\min \left(j, i^{\prime}-s\right)} .
$$


Here are the explicit results to order 4:

$$
\begin{aligned}
F_{0,0}= & 1, \quad F_{1,0}=\frac{\gamma_{1}}{\beta_{1}}-\frac{\gamma_{0}}{\beta_{0}}, F_{1,1}=\frac{\gamma_{0}}{\beta_{0}} \\
F_{2,0}= & \grave{\gamma}_{2}-\frac{\gamma_{0}}{\beta_{0}}+\frac{\gamma_{0} \beta_{2}}{\beta_{1}^{2}} \\
F_{2,1}= & \left(\frac{\gamma_{1}}{\beta_{1}}-\frac{\gamma_{0}}{\beta_{0}}\right)\left(1+\frac{\gamma_{0}}{\beta_{0}}\right)+\frac{\gamma_{0}}{\beta_{0}} \\
F_{2,2}= & \frac{1}{2} \frac{\gamma_{0}}{\beta_{0}}\left(1+\frac{\gamma_{0}}{\beta_{0}}\right) \\
F_{3,0}= & \grave{\gamma_{3}}+\left(\frac{\gamma_{1}}{\beta_{1}}-\frac{\gamma_{0}}{\beta_{0}}\right)\left(\frac{\gamma_{0}}{\beta_{0}}+1\right)\left(\frac{\beta_{0} \beta_{2}}{\beta_{1}^{2}}-1\right) \\
& -\frac{\gamma_{0}}{2 \beta_{0}}+\frac{\gamma_{0} \beta_{0} \beta_{3}}{2 \beta_{1}^{3}}, \\
F_{3,1}= & \left(2+\frac{\gamma_{0}}{\beta_{0}}\right)\left(\grave{\gamma}_{2}+\frac{\gamma_{0} \beta_{2}}{\beta_{1}^{2}}\right) \\
& +\left(1+\frac{\gamma_{0}}{\beta_{0}}\right)\left(\frac{\gamma_{1}}{\beta_{1}}-2 \frac{\gamma_{0}}{\beta_{0}}\right) \\
F_{3,3}= & \frac{1}{6} \frac{\gamma_{0}}{\beta_{0}}\left(1+\frac{\gamma_{0}}{\beta_{0}}\right)\left(2+\frac{\gamma_{0}}{\beta_{0}}\right) \\
F_{3,2}= & \left(\frac{\gamma_{1}}{\beta_{1}}-\frac{\gamma_{0}}{\beta_{0}}\right)\left(1+\frac{\gamma_{0}}{\beta_{0}}\right)\left(1+\frac{\gamma_{0}}{2 \beta_{0}}\right) \\
& \left.+\frac{\gamma_{0}}{\beta_{0}}\right), \\
& (95) \\
& (9)
\end{aligned}
$$

These coefficients $F_{i, j}$ satisfy the recursive relation

$$
F_{i, j}=\sum_{s=0}^{i-j} F_{i-j, s} \mathcal{R}(s, i, j),
$$

where $\mathcal{R}$ is a function of three non-negative integers, i.e.,

$$
\mathcal{R}(s, i, j)=\sum_{p=0}^{j} \sum_{t=s}^{j-p} \frac{\prod_{l=0}^{p-1}\left(\frac{\gamma_{0}}{\beta_{0}}+l\right)}{p !} \bigsqcup_{1}^{s} \frac{1}{t} \bigsqcup_{0}^{i-j} 1_{j-p-t} .
$$

The two square-cup operators, $\bigsqcup_{1}^{k} 1_{p}$ and $\bigsqcup_{0}^{i-j} 1_{j-t-p}$, are standard. Their expression can be found in Eqs. (A9) and (A12). For $i=j$ and $i=j+1$, Eq. (96) gives

$$
\begin{aligned}
F_{j, j}= & \frac{1}{j !} \prod_{l=0}^{j-1}\left(\frac{\gamma_{0}}{\beta_{0}}+l\right), \\
F_{j+1, j}= & \left(\frac{\gamma_{1}}{\beta_{1}}-\frac{\gamma_{0}}{\beta_{0}}\right) \prod_{l=1}^{j}\left(1+\frac{\gamma_{0}}{l \beta_{0}}\right) \\
& +\frac{\gamma_{0}}{\beta_{0}} \sum_{k=1}^{j} \frac{1}{k} \prod_{l=1}^{j-k}\left(1+\frac{\gamma_{0}}{l \beta_{0}}\right) .
\end{aligned}
$$

Truncating Eq. (90) to a finite order gives a new expression for quark masses, i.e.,

$$
m_{q}=m_{q n} L^{\frac{\gamma_{0}}{\beta_{0}}} \sum_{i=0}^{N-1}\left(\frac{\beta_{1}}{\beta_{0}^{2}} L\right)^{i} \sum_{j=0}^{i} F_{i, j} \ln ^{j} L .
$$

To leading order, it is simply

$$
m_{q}=m_{q n} L^{\gamma_{0} / \beta_{0}} \text {. }
$$

The next to leading order is

$$
m_{q}=m_{q n} L^{\frac{\gamma_{0}}{\beta_{0}}}\left[1+\frac{\beta_{1} \gamma_{0}}{\beta_{0}^{3}} L\left(\ln L+\frac{\beta_{0} \gamma_{1}}{\beta_{1} \gamma_{0}}-1\right)\right] \text {. }
$$

And the still next to leading reads

$$
\begin{aligned}
& m_{q}=m_{q n} L^{\frac{\gamma_{0}}{\beta_{0}}}\left\{1+L^{*}\left[\frac{\gamma_{0}}{\beta_{0}} \ln L+\frac{\gamma_{1}}{\beta_{1}}-\frac{\gamma_{0}}{\beta_{0}}\right]\right. \\
& +\frac{L^{* 2}}{2}\left[\left(\frac{\gamma_{0}}{\beta_{0}}+\frac{\gamma_{0}^{2}}{\beta_{0}^{2}}\right) \ln ^{2} L+2\left(\frac{\gamma_{1}}{\beta_{1}}+\frac{\gamma_{0} \gamma_{1}}{\beta_{0} \beta_{1}}-\frac{\gamma_{0}^{2}}{\beta_{0}^{2}}\right) \ln L\right. \\
& \left.\left.+\frac{\beta_{0} \gamma_{2}+\beta_{2} \gamma_{0}}{\beta_{1}^{2}}+\left(\frac{\gamma_{1}}{\beta_{1}}-\frac{\gamma_{0}}{\beta_{0}}\right)^{2}-\frac{\gamma_{1}}{\beta_{1}}-\frac{\gamma_{0}}{\beta_{0}}\right]\right\}
\end{aligned}
$$

Presently, this process can go to the fourth order according to the expansion coefficients listed in Eq. (95):

$$
\begin{aligned}
& m_{q}=m_{q n} L^{\frac{\gamma_{0}}{\beta_{0}}}\left[1+L^{*}\left(F_{1,0}+F_{1,1} \ln L\right)+\right. \\
& L^{* 2}\left(F_{2,0}+F_{2,1} \ln L+F_{2,2} \ln ^{2} L\right)+L^{* 3}\left(F_{3,0}\right. \\
& \left.\left.+F_{3,1} \ln L+F_{3,2} \ln ^{2} L+F_{3,3} \ln ^{3} L\right)\right] .
\end{aligned}
$$

Now we have three mass formulas, respectively, in Eqs. (100), (77), and (78). To use Eq. (100), we need to know $\Lambda$ which has been listed in Tab. I, and we take the first row in the table for each $\Lambda_{n}$ which has been determined from Eq. (32). To use Eqs. (77) and (78), however, we need to know not only $\Lambda$, but also the corresponding $\alpha$. So we naturally use the $\alpha$ expression in Eq. (5) for the mass formula in Eq. (77), and choose the expression in Eq. (14) for the mass formula in Eq. (78), in the present numerical calculations. The next step, we should use an initial condition to determine the mass scale $m_{q n}$. For $q=t, b$, and $c$, the initial condition is

$$
m_{q}\left(\bar{m}_{q}\right)=\bar{m}_{q}
$$

with $\bar{m}_{t}=172.76 \mathrm{GeV}, \bar{m}_{b}=4.18 \mathrm{GeV}, \bar{m}_{c}=1.27 \mathrm{GeV}$ [17]. And for $s$ quark, the initial condition is [17]

$$
m_{s}(2 \mathrm{GeV})=0.093 \mathrm{GeV} .
$$

The mass scale $m_{q n}$, calculated from these initial conditions and the three mass formulas in Eqs. (100), (77), and (78), are listed, respectively, in Tab. II, III, and IV.

Comparing the results in these tables, one can find that the leading mass scale $m_{q n}$ from the new mass formula with the new coupling expression is generally closer to the order- 4 value than the two previous formulas. Figure 4 shows that the new expression converges indeed very rapidly. The faster convergence speed can also been seen from Fig. 3(b) and 3(c), where the leading masses of $s$, $c, b$, and $t$ from the new expression (dashed line) are compared with that from the previous formulas at leading order (dotted line) and the order-4 average of the three formulas (solid line). 
TABLE II. QCD mass scale $m_{q n}(q=s, c, b, t$, and $n=3-6)$ from the new expression in Eq. (90) with Eq. (28).

\begin{tabular}{cccccc}
\hline flavor & $\mathrm{GeV}$ & $N=1$ & $N=2$ & $N=3$ & $N=4$ \\
\hline \multirow{4}{*}{ strange } & $m_{s 3}$ & .166190 & .165242 & .168878 & .168611 \\
& $m_{s 4}$ & .180222 & .177364 & .181712 & .181626 \\
& $m_{s 5}$ & .205088 & .198502 & .203271 & .203277 \\
& $m_{s 6}$ & .249948 & .237593 & .243259 & .243319 \\
\hline \multirow{4}{*}{ charm } & $m_{c 3}$ & 2.00329 & 1.92027 & 1.97396 & 1.97457 \\
& $m_{c 4}$ & 2.17243 & 2.06114 & 2.12398 & 2.12698 \\
& $m_{c 5}$ & 2.47218 & 2.30678 & 2.37598 & 2.38054 \\
& $m_{c 6}$ & 3.01292 & 2.76106 & 2.84339 & 2.84946 \\
\hline \multirow{3}{*}{ bottom } & $m_{b 3}$ & 8.69277 & 8.87212 & 8.98989 & 8.96995 \\
& $m_{b 4}$ & 9.42671 & 9.52301 & 9.67308 & 9.66232 \\
& $m_{b 5}$ & 10.7274 & 10.6579 & 10.8208 & 10.8142 \\
& $m_{b 6}$ & 13.0738 & 12.7568 & 12.9494 & 12.9443 \\
\hline \multirow{4}{*}{ top } & $m_{t 3}$ & 544.982 & 569.943 & 570.725 & 569.636 \\
& $m_{t 4}$ & 590.995 & 611.756 & 614.098 & 613.605 \\
& $m_{t 5}$ & 672.539 & 684.662 & 686.958 & 686.752 \\
& $m_{t 6}$ & 819.645 & 819.492 & 822.098 & 822.029 \\
\hline
\end{tabular}

TABLE III. QCD mass scale $m_{q n}(q=s, c, b, t$, and $n=3-6)$ from the previous expression in Eq. (77) with Eq. (5).

\begin{tabular}{cccccc}
\hline flavor & $\mathrm{GeV}$ & $N=1$ & $N=2$ & $N=3$ & $N=4$ \\
\hline \multirow{4}{*}{ strange } & $m_{s 3}$ & .195152 & .170540 & .170165 & .168681 \\
& $m_{s 4}$ & .213423 & .182521 & .182541 & .180981 \\
& $m_{s 5}$ & .241942 & .204203 & .204186 & .202456 \\
& $m_{s 6}$ & .291731 & .244484 & .244388 & .242328 \\
\hline \multirow{4}{*}{ charm } & $m_{c 3}$ & 2.44972 & 1.98781 & 2.00490 & 1.96575 \\
& $m_{c 4}$ & 2.67908 & 2.12746 & 2.15072 & 2.10908 \\
& $m_{c 5}$ & 3.03708 & 2.38019 & 2.40574 & 2.35934 \\
& $m_{c 6}$ & 3.66207 & 2.84970 & 2.87940 & 2.82398 \\
\hline \multirow{3}{*}{ bottom } & $m_{b 3}$ & 9.80497 & 9.08045 & 9.02424 & 8.99662 \\
& $m_{b 4}$ & 10.7230 & 9.71836 & 9.68058 & 9.65261 \\
& $m_{b 5}$ & 12.1558 & 10.8729 & 10.8284 & 10.7980 \\
& $m_{b 6}$ & 14.6574 & 13.0176 & 12.9605 & 12.9245 \\
\hline \multirow{4}{*}{ top } & $m_{t 3}$ & 576.004 & 574.469 & 572.311 & 572.199 \\
& $m_{t 4}$ & 629.932 & 614.826 & 613.936 & 613.920 \\
& $m_{t 5}$ & 714.109 & 687.865 & 686.733 & 686.767 \\
& $m_{t 6}$ & 861.064 & 823.551 & 821.944 & 822.018 \\
\hline
\end{tabular}

TABLE IV. QCD mass scale $m_{q n}(q=s, c, b, t$, and $n=3-6)$ from the previous expression in Eq. (78) with Eq. (14).

\begin{tabular}{cccccc}
\hline flavor & $\mathrm{GeV}$ & $N=1$ & $N=2$ & $N=3$ & $N=4$ \\
\hline \multirow{4}{*}{ strange } & $m_{s 3}$ & .195153 & .173371 & .170851 & .169346 \\
& $m_{s 4}$ & .213424 & .185883 & .183164 & .181736 \\
& $m_{s 5}$ & .241944 & .207836 & .204865 & .203312 \\
& $m_{s 6}$ & .291734 & .248729 & .245222 & .243350 \\
\hline \multirow{4}{*}{ charm } & $m_{c 3}$ & 2.44973 & 2.07294 & 2.01778 & 1.98684 \\
& $m_{c 4}$ & 2.67909 & 2.22255 & 2.16321 & 2.13220 \\
& $m_{c 5}$ & 3.03709 & 2.48504 & 2.41950 & 2.38534 \\
& $m_{c 6}$ & 3.66210 & 2.97398 & 2.89613 & 2.85508 \\
\hline \multirow{3}{*}{ bottom } & $m_{b 3}$ & 9.80494 & 9.10877 & 9.05075 & 9.00491 \\
& $m_{b 4}$ & 10.7229 & 9.76615 & 9.70307 & 9.66372 \\
& $m_{b 5}$ & 12.1558 & 10.9196 & 10.8526 & 10.8110 \\
& $m_{b 6}$ & 14.6574 & 13.0680 & 12.9905 & 12.9400 \\
\hline \multirow{4}{*}{ top } & $m_{t 3}$ & 575.996 & 573.817 & 573.485 & 572.004 \\
& $m_{t 4}$ & 629.924 & 615.230 & 614.818 & 613.853 \\
& $m_{t 5}$ & 714.099 & 687.890 & 687.659 & 686.732 \\
& $m_{t 6}$ & 861.056 & 823.235 & 823.123 & 821.969 \\
\hline
\end{tabular}

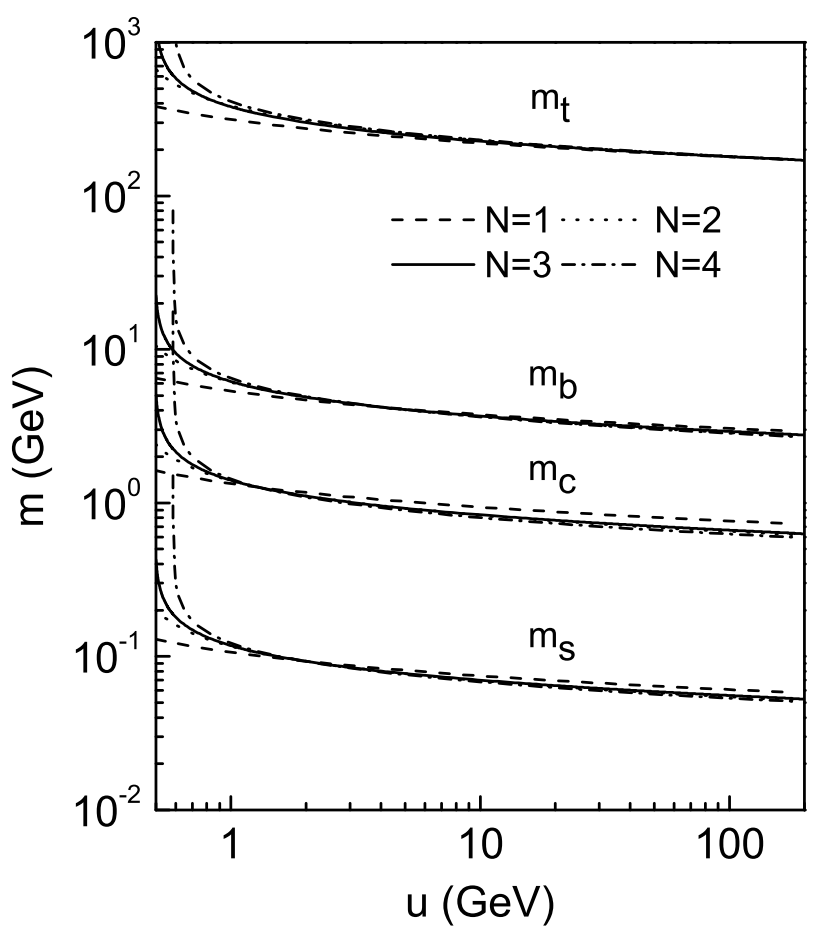

FIG. 4. The running masses of $t, b, c$, and $s$ quarks as functions of the RG point $u$, calculated by the new expression in Eq. (100) with $m_{q n}$ in Tab. II and $\Lambda$ in Tab. I, for the order $N$ from 1 to 4 .

\section{SUMMARY}

The renormalization group equations for both the QCD coupling and quark masses have been solved in detailed in a mathematically strict way. The general relation between the standard expansion coefficients and the beta and gamma functions are derived. It is accordingly proved that the fastest convergent expression can be obtained by simple substitution, which includes the lost known logarithmic terms and excludes incorrect terms. At the same time, quark masses can also been expressed as a direct series of the renormalization point.

It has also been shown that the original beta and gamma coefficients can be linearly re-combined to give matching-invariant coupling and quark masses. The concrete matching-invatiant beta and gamma coefficients are presented to order 4 , with a presently incorrect expression for $\beta_{2}$ corrected.

Numerical calculations based on the new experimental average $\alpha_{s}\left(M_{\mathrm{Z}}\right)$ has been performed to determined the QCD scale $\Lambda$ and quark mass scale $m_{q n}$. The results show that the new expressions for both the coupling and quark masses converge indeed much faster. 


\section{ACKNOWLEDGMENTS}

The authors would like to thank support from National Natural Science Foundation of China (Grant No. 11875052, 11575190, and 11135011).

\section{Appendix A: square cup operator}

For convenience in the mathematical derivations, we have introduced the square-cup operator $\bigsqcup$. The onedimensional square-cup operator, $\bigsqcup_{m}^{k}\left(a_{l}\right)_{i}$ or simply $\bigsqcup_{m}^{k} a_{i}$ for short, is a discrete functional of the three nonnegative inters $k, m$, and $i$ and a discrete function $a_{l}$. Its value is defined by

$$
\left(\sum_{i=m}^{\infty} a_{i} x^{i}\right)^{k}=\sum_{i=0}^{\infty}\left(\bigsqcup_{m}^{k} a_{i}\right) x^{i}
$$

Direct observation gives

$$
\bigsqcup_{m}^{0} a_{i}=\delta_{i, 0}, \bigsqcup_{m}^{1} a_{i<m}=0, \bigsqcup_{m}^{1} a_{i \geq m}=a_{i} .
$$

For $k \geq 2$, it is also not difficult to provide a general explicit expression

$$
\bigsqcup_{m}^{k} a_{i}=\left(\prod_{s=1}^{k-1} \sum_{p_{s}=m}^{i-(k-s) m-\varsigma_{s}^{p}}\right) a_{i-\varsigma_{k}^{p}} \prod_{r=1}^{k-1} a_{p_{r}}
$$

where

$$
\varsigma_{s}^{p} \equiv\left\{\begin{array}{ll}
\sum_{t=1}^{s-1} p_{t} & \text { if } s \geq 2 \\
0 & \text { otherwise }
\end{array} .\right.
$$

The meaning of $\varsigma_{k}^{p}$ is similar to this. Here are several special simple expressions:

$$
\begin{gathered}
\bigsqcup_{m}^{k>0} a_{0}=\delta_{m, 0} a_{0}^{k}, \bigsqcup_{m}^{k} a_{i<k m}=\bigsqcup_{m}^{k>\frac{i}{m}} a_{i}=0, \\
\bigsqcup_{m}^{k} a_{i<k m}=\bigsqcup_{m}^{k>\frac{i}{m}} a_{i}=0, \bigsqcup_{m}^{k>0} a_{k m}=a_{m}^{k} .
\end{gathered}
$$

If $a_{m} \neq 0$, the last equality of Eq. (A6) is also valid for $k=0$.

As an example, let's consider the Taylor coefficients of $[-\ln (1+x)]^{k}$. Integrating both sides of $1 /(1-x)=$ $\sum_{i=0}^{\infty} x^{i}$ gives $-\ln (1-x)=\sum_{i=1}^{\infty} x^{i} / i$, then

$$
[-\ln (1-x)]^{k}=\left[\sum_{i=1}^{\infty} \frac{1}{i} x^{i}\right]^{k}=\sum_{i=0}^{\infty}\left(\bigsqcup_{1}^{k} \frac{1}{i}\right) x^{i}
$$

where $\bigsqcup_{1}^{k} \frac{1}{i}$ is a short notation with the meaning

$$
\bigsqcup_{1}^{k} \frac{1}{i}=\bigsqcup_{1}^{k}\left(a_{0}=0, a_{l}=\frac{1}{l}\right)_{i}= \begin{cases}\delta_{i, 0} & \text { if } k=0 \\ a_{i} & \text { if } k=1 \\ \bigsqcup_{1}^{k \geq 2} \frac{1}{i} & \text { if } k \geq 2\end{cases}
$$

and

$$
\begin{aligned}
\bigsqcup_{1}^{k \geq 2} \frac{1}{i} & =\left(\prod_{s=1}^{k-1} \sum_{p_{s}=1}^{i-k+s-\varsigma_{s}^{p}}\right) \frac{1}{i-\varsigma_{k}^{p}} \prod_{r=1}^{k-1} \frac{1}{p_{r}} \\
= & \sum_{p_{1}=1}^{i-1} \frac{1}{p_{1}\left(i-p_{1}\right)} \text { if } k=2, \\
=\sum_{p_{1}=1}^{i-2} \sum_{p_{2}=1}^{i-1-p_{1}} \frac{1}{p_{1} p_{2}\left(i-p_{1}-p_{2}\right)} \text { if } k=3, &
\end{aligned}
$$

In the case of $a_{l}=1$, the multi-summation can be replaced by compact expressions, e.g.,

$$
\begin{aligned}
& \bigsqcup_{1}^{k \geq 2} 1_{i}=\bigsqcup_{1}^{k \geq 2}\left(a_{l}=1\right)_{i}=\frac{1}{(k-1) !} \prod_{t=1}^{k-1}(i-t), \\
& \bigsqcup_{0}^{k \geq 1} 1_{i}=\bigsqcup_{0}^{k \geq 1}\left(a_{l}=1\right)_{i}=\frac{(i+k-1) !}{i !(k-1) !}
\end{aligned}
$$

To have completeness for the square-cup operators $\bigsqcup_{1}^{k} 1_{i}$ and $\bigsqcup_{0}^{k} 1_{i}$, we further list

$$
\bigsqcup_{0}^{0} 1_{i}=\bigsqcup_{1}^{0} 1_{i}=\delta_{i, 0}, \bigsqcup_{1}^{1} 1_{0}=0, \bigsqcup_{1}^{1} 1_{i \geq 1}=1
$$

The two-dimensional extension of the square-cup operator, $\bigsqcup_{m, n}^{k}(f)_{i, j}$ or $\bigsqcup_{m, n}^{k} f_{i, j}$ for short, is defined by

$$
\left(\sum_{i=m}^{\infty} \sum_{j=n}^{i} f_{i, j} x^{i} y^{j}\right)^{k}=\sum_{i=0}^{\infty} \sum_{j=0}^{i}\left(\bigsqcup_{m, n}^{k} f_{i, j}\right) x^{i} y^{j} .
$$

Similarly we have

$$
\begin{gathered}
\bigsqcup_{m, n}^{0} f_{i, j}=\delta_{i, 0} \delta_{j, 0}, \bigsqcup_{m, n}^{1} f_{i<m, j}=0 \\
\bigsqcup_{m, n}^{1} f_{i, j<n}=0, \bigsqcup_{m, n}^{1} f_{i \geq m, j \geq n}=f_{i, j}
\end{gathered}
$$

and for $k \geq 2$, we have

$$
\bigsqcup_{m, n}^{k} f_{i, j}=\left(\prod_{s=1}^{k-1} \sum_{p_{s}=m}^{i-(k-s) m-\varsigma_{s}^{p}} \sum_{q_{s}=\sigma}^{p_{s}^{*}}\right) f_{i-\varsigma_{k}^{p}, j-\varsigma_{k}^{q}} \prod_{r=1}^{k-1} f_{p_{r}, q_{r}}
$$

where

$$
\begin{aligned}
p_{s}^{*} & \equiv \min \left[p_{s}, j-(k-s) n-\varsigma_{s}^{q}\right] \\
\sigma & \equiv \max \left(n, p_{s}+\varsigma_{s}^{p}-\varsigma_{s}^{q}-i+j\right) .
\end{aligned}
$$


Here are special simple cases:

$$
\begin{gathered}
\bigsqcup_{m, n}^{k} f_{0,0}=\delta_{m, 0} \delta_{n, 0} f_{0,0}^{k}, \bigsqcup_{m, n}^{k} f_{k m, k n}=f_{m, n}^{k}, \\
\bigsqcup_{m, n}^{k} f_{i<k m, j}=\bigsqcup_{m, n}^{k} f_{i, j<k n}=\bigsqcup_{m, n}^{k>\frac{i}{m}} f_{i, j}=\bigsqcup_{m, n}^{k>\frac{j}{n}} f_{i, j}=0 \\
\bigsqcup_{m, n}^{k} f_{i<j, j}=\bigsqcup_{m, n}^{k} f_{i, j>i}=0
\end{gathered}
$$

From Eqs. (A19) and (A20), we can write

$$
p_{s}+\sum_{t=1}^{s-1}\left(p_{t}-q_{t}\right)-i+j \geq q_{s} \geq p_{s} .
$$

If $j=i$, this means $q_{s}=p_{s}$. Therefore, the twodimensional square-cup operator reduces to the onedimensional case, i.e.,

$$
\bigsqcup_{m, n}^{k} f_{i, i}=\bigsqcup_{m}^{k}\left(a_{l}=f_{l, l}\right)_{i}
$$

If $f_{i, i}=1(i=0,1,2, \cdots)$, which is exactly the case for the expansion coefficients of the QCD coupling, one can check the following equality:

$$
\bigsqcup_{1,0}^{k} f_{i, i}=\bigsqcup_{1}^{k} 1_{i}
$$

The expression for $\bigsqcup_{1}^{k} 1_{i}$ has been listed in Eqs. (A12) and (A14). From the above equation, one can further prove

$$
\begin{gathered}
\sum_{k=0}^{i} \frac{\prod_{l=0}^{k-1}(z-l)}{k !} \bigsqcup_{1,0}^{k} f_{i, i}=\frac{1}{i !} \prod_{l=0}^{i-1}(z+l), \\
\sum_{i=0}^{j} \sum_{k=0}^{i} \frac{\prod_{l=0}^{k-1}(z-l)}{k !} \bigsqcup_{1}^{k} 1_{i}=\prod_{l=1}^{j}\left(1+\frac{z}{l}\right),
\end{gathered}
$$

where $z$ is an arbitrary number.

\section{Appendix B: Matching condition for the running coupling}

In the conventional $\overline{\mathrm{MS}}$, the strong coupling $\alpha(u)$ is not continuous at heavy quark masses. Suppose $\alpha$ is the coupling in the full $N_{\mathrm{f}}$-flavor theory while $\alpha^{*}$ belong to the effective theory with $N_{\mathrm{f}}-1$ flavors. Then they can be linked by the matching condition:

$$
\alpha^{*}=\alpha \sum_{i=0}^{\infty} \sum_{j=0}^{i} C_{i, j} \alpha^{i} \ln ^{j} \frac{m_{\mathrm{h}}^{2}}{u^{2}} .
$$

If the heavy quark threshold $m_{\mathrm{h}}$ is taken to be running, it should satisfy the RG equation for the quark mass, i.e.,

$$
\frac{\mathrm{d} \ln m_{\mathrm{h}}}{\mathrm{d} \ln u^{2}}=-\sum_{i=0}^{\infty} \gamma_{i} \alpha^{i+1}
$$

where the quark mass anomalous dimension $\gamma_{i}$ are given in Eq. (41). At the same time, $\alpha^{*}$ should also satisfies the $\mathrm{RG}$ equation for coupling, i..e,

$$
\frac{\mathrm{d} \alpha^{*}}{\mathrm{~d} \ln u^{2}}=-\sum_{k=0}^{\infty} \beta_{k}^{*} \alpha^{* k+2}
$$

where $\beta_{k}^{*}=\beta_{k}\left(N_{\mathrm{f}} \rightarrow N_{\mathrm{f}}-1\right)$. Substituting Eq. (B1) into this equation and taking into account Eq. (1), the left hand side of Eq. (B3) becomes

$$
\begin{aligned}
(L)= & -\sum_{i=0}^{\infty} \sum_{j=0}^{i}\left(\sum_{k=0}^{i-j}(i-k+1) \beta_{k} C_{i-k, j}\right) \alpha^{i+2} \ln ^{j} \frac{m_{\mathrm{h}}^{2}}{u^{2}} \\
& -2 \sum_{i=1}^{\infty} \sum_{j=0}^{i-1}\left(\sum_{k=0}^{i-j-1}(j+1) \gamma_{k} C_{i-k, j+1}\right) \alpha^{i+2} \ln ^{j} \frac{m_{\mathrm{h}}^{2}}{u^{2}} \\
& -\sum_{i=0}^{\infty} \sum_{j=0}^{i}(j+1) C_{i+1, j+1} \alpha^{i+2} \ln ^{j} \frac{m_{\mathrm{h}}^{2}}{u^{2}} .
\end{aligned}
$$

and the right hand side of Eq. (B3) is

$$
(R)=-\sum_{i=0}^{\infty} \sum_{j=0}^{i}\left(\sum_{k=0}^{i-j} \beta_{k}^{*} \bigsqcup_{0,0}^{k+2} C_{i-k, j}\right) \alpha^{i+2} \ln ^{j} \frac{m_{\mathrm{h}}^{2}}{u^{2}}
$$

Comparing the coefficients of $\alpha^{i+2} \ln ^{j}\left(m_{\mathrm{h}}^{2} / u^{2}\right)$ then gives

$$
\begin{aligned}
C_{i, j}= & \frac{1}{j} \sum_{k=0}^{i-j}\left(\beta_{k}^{*} \bigsqcup_{0,0}^{k+2}-(i-k) \beta_{k}\right) C_{i-k-1, j-1} \\
& -2 \sum_{k=0}^{i-j-1} \gamma_{k} C_{i-k-1, j}
\end{aligned}
$$

which determine all the coefficients $C_{i, j \neq 0}$. To know $C_{i, 0}$, one has to perform perturbative calculations [5, 18-20]. Here are the results for $C_{i, 0}$ to three-loop level in the modified minimum subtraction scheme

$$
\begin{aligned}
& C_{0,0}=1, \quad C_{1,0}=0, \quad C_{2,0}=11 / 72, \\
& C_{3,0}=\frac{575263}{124416}-\frac{82043}{27648} \zeta_{3}-\frac{2633}{31104} N_{\mathrm{f}} .
\end{aligned}
$$

With these values, Eq. (B6) gives

$$
\begin{aligned}
& C_{i, j}=0 \quad \text { if } j>i \\
& C_{i, j}=1 / 6^{i} \text { if } j=i,
\end{aligned}
$$


And for $j<i$, one has

$$
\begin{aligned}
C_{2,1}= & =11 / 24 \\
C_{3,2}= & =\frac{23}{192}-\frac{n}{36}, C_{3,1}=\frac{511}{288}-\frac{67}{576} n \\
C_{4,3}= & \frac{1597}{10368}+\frac{53}{1728} n-\frac{1}{324} n^{2} \\
C_{4,2}= & \frac{5317}{6912}-\frac{703}{5184} n-\frac{77}{20736} n^{2} \\
C_{4,1}= & -\frac{6865}{186624} n^{2}-\left(\frac{110779}{82944} \zeta_{3}-\frac{137801}{373248}\right) n \\
& +\frac{2751301}{165888} \zeta_{3}-\frac{7639841}{746496}
\end{aligned}
$$

Or explicitly, one has the matching condition

$$
\begin{aligned}
\frac{\alpha^{*}}{\alpha} & =1+\alpha t+\alpha^{2}\left(t^{2}+\frac{11}{4} t+\frac{11}{72}\right) \\
& +\alpha^{3}\left[t^{3}+\left(\frac{69}{16}-N_{\mathrm{f}}\right) t^{2}+\left(\frac{511}{48}-\frac{67}{96} N_{\mathrm{f}}\right) t\right. \\
& \left.+\frac{575263}{124416}-\frac{82043}{27648} \zeta_{3}-\frac{2633}{31104} N_{\mathrm{f}}\right]+\cdots
\end{aligned}
$$

where $t=(1 / 6) \ln \left(m_{\mathrm{h}}^{2} / u^{2}\right)$.

If one would like to use a RG-invariant mass in Eq. (B1), then the last term containing $\gamma_{k}$ in Eq. (B6) should vanish. Accordingly, the matching condition becomes

$$
\begin{aligned}
\frac{\alpha^{*}}{\alpha} & =1+\alpha t+\alpha^{2}\left(t^{2}+\frac{19}{4} t+\frac{11}{72}\right) \\
& +\alpha^{3}\left[t^{3}-\frac{131}{16} t^{2}+\left(\frac{393}{16}-\frac{281}{288} N_{\mathrm{f}}\right) t\right. \\
& \left.+\frac{575263}{124416}-\frac{82043}{27648} \zeta_{3}-\frac{2633}{31104} N_{\mathrm{f}}\right]+\cdots
\end{aligned}
$$

In some cases, it would be more convenient to write the matching condition in Eq. (B1) in the form [21]

$$
\alpha=\alpha^{*} \sum_{i=0}^{\infty} \sum_{j=0}^{i} C_{i, j}^{*} \alpha^{* i} \ln ^{j} \frac{m_{\mathrm{h}^{2}}}{u^{2}}
$$

Replacing the $\alpha^{*}$ here with the right hand side of Eq. (B1) gives the relation between the coefficients $C_{i, j}^{*}$ and $C_{i, j}$ :

$$
\sum_{k=0}^{i} \sum_{l=\max (0, j-k)}^{\min (j, i-k)} C_{k, j-l}^{*} \bigsqcup_{0,0}^{k+1} C_{i-k, l}=\delta_{i, 0} \delta_{j, 0}
$$

Here are the solution to order 4:

$$
\begin{aligned}
C_{0,0}^{*}= & 1, C_{1,0}^{*}=0, C_{i, i}^{*}=\left(-C_{1,1}\right)^{i}, \\
C_{2,0}^{*}= & -C_{2,0}, C_{2,1}^{*}=-C_{2,1}, \\
C_{3,0}^{*}= & -C_{3,0}, C_{3,1}^{*}=-C_{3,1}+5 C_{2,0} C_{1,1}, \quad(\mathrm{~B} 14 \mathrm{~b}) \\
C_{3,2}^{*}= & -C_{3,2}+5 C_{1,1} C_{2,1}, C_{4,0}^{*}=-C_{4,0}+3 C_{2,0}^{2}, \\
C_{4,1}^{*}= & -C_{4,1}+6\left(C_{3,0} C_{1,1}+C_{2,0} C_{2,1}\right), \\
C_{4,2}^{*}= & -C_{4,2}+3 C_{2,1}^{2}-21 C_{2,0} C_{1,1}^{2} \\
& +6\left(C_{1,1} C_{3,1}+C_{2,0} C_{2,2}\right), \\
C_{4,3}^{*}= & -C_{4,3}-21 C_{2,1} C_{1,1}^{2}+6\left(C_{1,1} C_{3,2}+C_{2,1} C_{2,2}\right) .
\end{aligned}
$$

\section{Appendix C: Matching-invariant coupling}

In the traditional minimum subtraction scheme $(\overline{\mathrm{MS}})$, the strong coupling $\alpha(u)$ as a function of the renormalization point $u$ is not continuous at the quark masses. In this section, let's derive a matching-invariant coupling by absorbing loop effects into the $\overline{\mathrm{MS}}$ definition and give the corresponding $\beta$ function to four-loop level.

Suppose the new coupling $\alpha^{\prime}$ is connected to the original coupling $\alpha$ by

$$
\alpha^{\prime}=\sum_{i=0}^{\infty} a_{i} \alpha^{i+1}
$$

then,

$$
\begin{aligned}
\alpha^{\prime *} & =\sum_{i=0}^{\infty} a_{i}^{*} \alpha^{* i+1}=\sum_{i=0}^{\infty} a_{i}^{*}\left(\sum_{j=0}^{\infty} C_{j, 0} \alpha^{j+1}\right)^{i+1} \\
& =\sum_{i=0}^{\infty} a_{i}^{*} \sum_{j=0}^{\infty}\left(\bigsqcup_{0}^{i+1} C_{j, 0}\right) \alpha^{i+j+1} \\
& =\sum_{i=0}^{\infty}\left[\sum_{k=0}^{i} a_{k}^{*} \bigsqcup_{0}^{k+1} C_{i-k, 0}\right] \alpha^{i+1}
\end{aligned}
$$

Comparing the coefficients of $\alpha^{i+1}$ in the equality $\alpha^{\prime *}=$ $\alpha^{\prime}$ leads to

$$
a_{i}=\sum_{k=0}^{i} a_{k}^{*} \bigsqcup_{0}^{k+1} C_{i-k, 0}
$$

Assume

$$
a_{i}=\sum_{j=0}^{i} a_{i, j} n^{j}
$$

then $a_{k}^{*}=\sum_{j=0}^{k} a_{k, j}(n-1)^{j}$. Substituting into Eq. (C3) then gives

$$
\sum_{k=0}^{i}\left[a_{i, k} n^{k}-\left(\bigsqcup_{0}^{k+1} C_{i-k, 0}\right) \sum_{j=0}^{k} a_{k, j}(n-1)^{j}\right]=0
$$


whose solution is

$$
\begin{aligned}
a_{0}= & a_{0,0}, \quad a_{1}=a_{1,0}, \quad a_{2}=a_{2,0}+\frac{11}{72} a_{0,0} n, \quad \\
a_{3}= & a_{3,0}+\left[\left(\frac{7037}{1536}-\frac{82043}{27648} \zeta_{3}\right) a_{0,0}+\frac{11}{36} a_{1,0}\right] n \\
& -\frac{2633}{62208} a_{0,0} N_{\mathrm{f}}^{2}, \cdots
\end{aligned}
$$

To definitely fix the new coupling, one needs to choose $a_{i, 0}$. The simplest non-trivial choice would be

$$
a_{i, 0}=\delta_{i, 0}= \begin{cases}1 & \text { if } i=0 \\ 0 & \text { otherwise }\end{cases}
$$

With this convention, one has

$$
\begin{aligned}
& a_{0}=1, \quad a_{1}=0, \quad a_{2}=(11 / 72) n, \\
& a_{3}=a_{3,1} n-\frac{2633}{62208} n^{2}, \cdots
\end{aligned}
$$

where $a_{3,1}=7037 / 1536-82043 \zeta_{3} / 27648 \approx 1.014382432$. Then the new matching-invariant coupling is

$$
\alpha^{\prime}=\alpha+\frac{11}{72} n \alpha^{3}+\left(a_{3,1}-\frac{2633}{62208} n\right) n \alpha^{4}+\cdots
$$

The inverse relation of Eq. (C1) is

$$
\alpha=\sum_{j=0}^{\infty} a_{j}^{\prime} \alpha^{j+1} .
$$

Replacing the $\alpha^{\prime}$ with the right hand side of Eq. (C1), and then comparing coefficients will give equations to determine the coefficients $a_{j}^{\prime}$ :

$$
\sum_{k=0}^{j} a_{k}^{\prime} \bigsqcup_{0}^{k+1} a_{j-k}=\delta_{j, 0}, \quad j=0,1,2, \cdots
$$

whose solution is

$$
\begin{aligned}
& a_{0}^{\prime}=\frac{1}{a_{0}}, a_{1}^{\prime}=-\frac{a_{1}}{a_{0}^{3}}, a_{2}^{\prime}=-\frac{a_{2}}{a_{0}^{4}}+2 \frac{a_{1}^{2}}{a_{0}^{5}}, \\
& a_{3}^{\prime}=-a_{3} / a_{0}^{5}+5 a_{1} a_{2} / a_{0}^{6}-5 a_{1}^{3} / a_{0}^{7}, \cdots
\end{aligned}
$$

On application of the $a_{i}$ values in Eq. (C8), these becomes $a_{0}^{\prime}=1, a_{1}^{\prime}=0, a_{2}^{\prime}=-\frac{11}{72} n, a_{3}^{\prime}=a_{3,1}^{\prime} n+\frac{2633}{62208} n^{2}, \cdots$

where $a_{3,1}^{\prime}=-7037 / 1536+(82043 / 27648) \zeta_{3} \approx$ -1.014382432 .
To transform a thermodynamic potential expressed with terms like $\alpha^{i} \ln ^{j} \alpha$, one also needs to know how to express $\ln \alpha$ with $\alpha^{\prime}$ and $\ln \alpha^{\prime}$. Here is the result:

$$
\ln \alpha=\ln \alpha^{\prime}+\sum_{j=1}^{\infty}\left(\sum_{k=1}^{j} \frac{(-1)^{k-1}}{k} \bigsqcup_{1}^{k} a_{j}^{\prime}\right) \alpha^{\prime j} .
$$

To three-loop level and with Eq. (C8), one explicitly has

$$
\alpha=\alpha^{\prime}-\frac{11}{72} n \alpha^{\prime 3}+n\left(a_{3,1}^{\prime}+\frac{2633}{62208} n\right) \alpha^{\prime 4}
$$

and

$$
\ln \alpha=\ln \alpha^{\prime}-\frac{11}{72} n \alpha^{\prime 2}+n\left(a_{3,1}^{\prime}+\frac{2633}{62208} n\right) \alpha^{\prime 3} .
$$

The renormalization equation for $\alpha^{\prime}$ is

$$
\frac{d \alpha^{\prime}}{d \ln u^{2}}=-\sum_{i=0}^{\infty} \beta_{i}^{\prime} \alpha^{i+2}
$$

The primed beta coefficients $\beta_{i}^{\prime}$ can be obtained by substituting Eq. (C1) into Eq. (C17), applying Eqs. (C17) and (1), and then comparing coefficients:

$$
\sum_{k=0}^{i}\left[\beta_{k}^{\prime} \bigsqcup_{0}^{k+2} a_{i-k}-(k+1) a_{k} \beta_{i-k}\right]=0,
$$

namely, $\beta_{i}^{\prime}$ are given by the recursive relation

$$
\beta_{i}^{\prime}=\sum_{k=0}^{i}(k+1) a_{k} \beta_{i-k}-\sum_{k=0}^{i-1} \beta_{k}^{\prime} \bigsqcup_{0}^{k+2} a_{i-k} .
$$

On application of Eqs. (C8a) and (C8b), one immediately has the explicit expressions:

$$
\begin{aligned}
\beta_{0}^{\prime} & =\beta_{0}=\frac{11}{4}-\frac{n}{6}, \beta_{1}^{\prime}=\beta_{1}=\frac{51}{8}-\frac{19}{24} n, \quad(\mathrm{C} 20 a) \\
\beta_{2}^{\prime} & =\beta_{2}+a_{2} \beta_{0}-a_{1}\left(\beta_{1}+a_{1} \beta_{0}\right) \\
& =\frac{2857}{128}-\frac{4549}{1162} n+\frac{79}{1152} n^{2}, \\
\beta_{3}^{\prime} & =\beta_{3}+2 a_{3} \beta_{0}-2 a_{1} \beta_{2}+a_{1}^{2} \beta_{1}+4 a_{1}^{3} \beta_{0}-6 a_{1} a_{2} \beta_{0} \\
& =\beta_{3}^{(0)}+\beta_{3}^{(1)} N_{\mathrm{f}}+\beta_{3}^{(2)} N_{\mathrm{f}}^{2}+\frac{23}{1152} N_{\mathrm{f}}^{3}, \quad(\mathrm{C} 20 c)
\end{aligned}
$$

with $\beta_{3}^{(0)}=149753 / 1536+891 \zeta_{3} / 64 \approx$ $114.2303287, \beta_{3}^{(1)}=-66733 / 82944-318179 \zeta_{3} / 18432 \approx$ $-21.55484044, \quad \beta_{3}^{(2)}=-68767 / 124416+$ $35977 \zeta_{3} / 27648 \approx 1.011459984$.

It should be mentioned that a different expression for $\beta_{2}^{\prime}$ was previously given in Ref. [16]. The error was caused by the fact that a wrong value for $C_{2}$ was quoted there [18]. 
[1] S. Schramm, W. Greiner, and E. Stein, Quantum Chromodynamics, Springer, 2nd edition, 2002.

[2] G. X. Peng, Europhys. Lett. 72, 69 (2005); Nucl. Phys. A 747, 75-83 (2005).

[3] E.S. Fraga and P. Romatschke, Phys. Rev. D 71 (2005) 105014; E.S. Fraga, R.D. Pisarski, and J. SchaffnerBielich, Phys. Rev. D 63 R121702 (2001).

[4] S. Eidelman et al. [Particle Data Group Collabration], Phy. Lett. B 592, 1 (2004).

[5] K.G. Chetyrkin, B.A. Kniehl, and M. Steinhauser, Phys. Rev. Lett. 79, 2184 (1997).

[6] G. 't Hooft, Nucl. Phys. B 61, 455 (1973);

[7] W.A. Bardeen, A.J. Buras, D.W. Duke, and T. Muta, Phys. Rev. D 18, 3998 (1978).

[8] G. X. Peng, Phys. Lett. B 634, 413 (2006).

[9] D. J. Gross and F. Wilczek, Phys. Rev. Lett. 30, 1343 (1973); H. D. Politzer, Phys. Rev. Lett. 30, 1346 (1973).

[10] W.E. Caswell, Phys. Rev. Lett. 33, 244 (1974); D.R.T. Jones, Nucl. Phys. B 75, 531 (1974). E.S. Egorian, O.V. Tarasov, Theor. Mat. Fiz. 41, 26 (1979).

[11] O.V. Tarasov, A.A. Vladimirov, A.Yu. Zharkov, Phys. Lett. B 93, 429 (1980); S.A. Larin and J.A.M. Vermaseren, Phys. Lett. B 303, 334 (1993).

[12] T. van Ritbergen, J.A.M. Vermaseren, and S.A. Larin,
Phys. Lett. B 400, 379 (1997).

[13] A. Buras, Rev. Mod. Phys. 52199 (1980).

[14] E. Gardi, G. Grunberg, and M. Karliner, JHEP 9807007 (1998).

[15] S. D. Bass, R.J. Crewther, F.M. Steffens, and A.W. Thomas, Phys. Rev. D 68096005 (2003).

[16] W.J. Marciano, Phys. Rev. D 29580 (1984).

[17] P.A. Zyla et al, [Particle Data Group 2020] Prog. Theor. Exp. Phys. 2020, 083 C01 (2020).

[18] W. Wetzel, Nucl. Phys. B 196, 259 (1982); W. Bernreuther and W. Wetzel, Nucl. Phys. B 197, 228 (1982); Erratum-ibid. B 513, 758 (1998); W. Bernreuther, Ann. Phys. (N.Y.) 151, 127 (1983); Z. Phys. C 20, 331 (1983).

[19] S.A. Larin, T. van Ritbergen, and J.A.M. Vermaseren, Nucl. Phys. B 438, 278 (1995).

[20] B. A. Kniehl, A.V. Kotikov, A. I. Onishchenko, and O. L. Veretin, Phys. Rev. Lett. 97, 042001 (2006).

[21] G. Rodrigo, A. Pich, A. Santamria, Phys. Lett. B 424, 367 (1998).

[22] K.G. Chetyrkin, Phys. Lett. B 404, 161 (1997).

[23] J.A.M. Vermaseren, S.A. Larin, and T. van Ritbergen, Phys. Lett. B 405, 327 (1997).

[24] K.G. Chetyrkin, B.A. Kniehl, and M. Steinhauser, Nucl. Phys. B 510, 61 (1998). 